\title{
Structural and Magnetic Properties of Nickel Antimony Ferrospinels
}

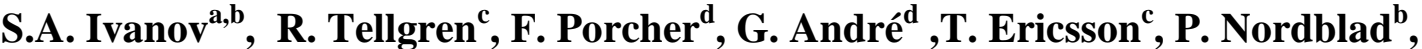 N. Sadovskaya ${ }^{\text {a }}$, G. Kaleva ${ }^{a}$, E. Politova ${ }^{\text {a }}$, M. Baldini ${ }^{\mathrm{e}}$, C. Sun ${ }^{\mathrm{f}}$, D. Arvanitis ${ }^{\mathrm{g}}$, P. Anil Kumar ${ }^{\mathrm{b}}$, and R. Mathieu ${ }^{\mathrm{b}, *}$}

a -Center of Materials Science, Karpov' Institute of Physical Chemistry, Moscow, 105064, Russia b -Department of Engineering Sciences, Uppsala University, Box 534, 75121 Uppsala, Sweden c -Department of Chemistry, Uppsala University, Box 538, 75121 Uppsala, Sweden d -Laboratoire Leon Brillouin, Saclay, France e -HPSynC, Carnegie Institution of Washington, Argonne, IL 60439, USA f -X-ray Science Division, Advanced Photon Source, Argonne National Laboratory, Argonne, IL 60439, USA g -Department of Physics and Astronomy, Uppsala University, Box 516, 75120 Uppsala, Sweden

*Tel.: +46-(0)18 47172 33; Fax: +46-(0)18 4713270

email address: roland.mathieu@angstrom.uu.se

\begin{abstract}
Spinel-type compounds of Fe-Ni-Sb-O system were synthesized as polycrystalline powders. The crystal and magnetic properties were investigated using X-ray and neutron powder diffraction, Mössbauer and X-ray absorption spectroscopy and magnetization measurements. The samples crystallize in the cubic system, space group Fd $-3 \mathrm{~m}$. The distribution of cations between octahedral and tetrahedral sites was refined from the diffraction data sets using constraints imposed by the magnetic, Mössbauer and EDS results and the ionic radii. The cation distribution and the temperature dependence of the lattice parameter $(\boldsymbol{a})$ and the oxygen positional parameter $(\boldsymbol{u})$ were obtained. A chemical formula close to $\mathrm{Fe}_{0.8} \mathrm{Ni}_{1.8} \mathrm{Sb}_{0.4} \mathrm{O}_{4}$ was determined, with $\mathrm{Sb}^{5+}$ cations occupying octahedral sites, and $\mathrm{Fe}^{3+}$ and $\mathrm{Ni}^{2+}$ occupying both tetrahedral and octahedral sites. $\mathrm{Fe}^{3+}$ mainly (85/15 ratio) occupy tetrahedral sites, and conversely $\mathrm{Ni}^{2+}$ mainly reside on octahedral ones.

The magnetic unit cell is the same as the crystallographic one, having identical symmetry relations. The results indicate that the compounds have a collinear ferrimagnetic structure with antiferromagnetic coupling between the tetrahedral (A) and octahedral (B) sites. Uniquely, the temperature dependence of the net magnetization of this rare earth free ferrimagnet exhibits a compensation point.
\end{abstract}

Keywords: Oxides; Neutron scattering and diffraction; Crystal structure; Magnetic properties 


\section{Introduction}

The search for novel multifunctional materials is an active field in material science. Within this field of research, transition metal oxides have been under scrutiny for more than twenty years due to diverse collective properties arising from the interaction between the transition metal and oxygen ions and its strong dependence on bond lengths and angles [1-3]. Consequently, a large number of materials showing different physical effects can be found within the same structural family. Perovskites are most investigated because of their structural flexibility that allows one to combine layers of selected materials with very different properties to design multifunctional structures. Besides perovskites, spinel ferrites are also very versatile with many degrees of freedom to engineer the physical properties. Magnetic ferrites with the spinel structure of the type $\mathrm{MFe}_{2} \mathrm{O}_{4}$ (M is a divalent cation) are since long of great importance in science and industry owing to their diverse magnetic and electrical properties [4-6]. Their applications range from simple permanent magnets to sophisticated devices for the electro-electronic industry. Some interesting applications, which include recent scientific and technological advances, are found in computer peripherals, telecommunication equipment, electronic and microwave devices, magnetic media used in computers, recording devices and magnetic cards.

Many composite multiferroic thin films use the soft-magnetic ferrite $\mathrm{NiFe}_{2} \mathrm{O}_{4}$ as the magnetic component [7]. However, spinel oxides seem neither to favor conventional ferroelectricity nor the so far rarely observed magnetically driven ferroelectricity seen in e.g. $\mathrm{CoCr}_{2} \mathrm{O}_{4}, \mathrm{CdV}_{2} \mathrm{O}_{4}$ and $\mathrm{FeV}_{2} \mathrm{O}_{4}[8]$.

The electrical and magnetic properties of ferrites are governed by parameters such as processing conditions, sintering temperature and time as well as chemical composition and structural features including the distribution of cations over the tetrahedral and octahedral sublattices [9]. The structure of cubic spinel ferrites is based on cubic close-packing of anions with the cations located on $1 / 8$ of the tetrahedral and $1 / 2$ of the octahedral sites. The general formula for the cationic distribution is $\left(A_{1-\gamma} B \gamma\right)\left[B_{2-\gamma} A \gamma\right] O_{4}$; where $\gamma$ is the degree of inversion (0 $<\gamma<1.0)$ and parentheses and square brackets indicate tetrahedral and octahedral sites, respectively. Without inversion, the chemical formula becomes $(A)\left[B_{2}\right] \mathrm{O}_{4}$, or more simply $\mathrm{AB}_{2} \mathrm{O}_{4}$, in which $\mathrm{A}$ and $\mathrm{B}$ refers to tetrahedral and octahedral sites, respectively. The cation distribution between octahedral and tetrahedral sites is crucial, since it determines the magnetic and electrical properties [10]. The compounds are characterized by two structural parameters: the lattice constant $\boldsymbol{a}$ and the oxygen parameter $\boldsymbol{u}[10,11]$. 
Apart from the classic work of Blasse [12,13], studies on the substitution of $\mathrm{Fe}^{3+}$ in

$\mathrm{NiFe}_{2} \mathrm{O}_{4}$ with diamagnetic pentavalent cations, such as $\mathrm{Sb}^{5+}$, are scarce in the literature. The process of incorporating $\mathrm{Sb}^{5+}$ in $\mathrm{NiFe}_{2} \mathrm{O}_{4}$ requires using excess $\mathrm{Ni}^{2+}$ cations to substitute $\mathrm{Fe}^{3+}$ ions thereby maintaining the charge balance in the formula $\mathrm{Ni}_{1+2 \mathrm{x}} \mathrm{Sb}_{\mathrm{x}} \mathrm{Fe}_{2-3 \mathrm{x}} \mathrm{O}_{4}$. The general cationic distribution proposed by Blasse [12] for this system may be represented as

$$
\left(\mathrm{Ni}^{2+}{ }_{\delta} \mathrm{Fe}^{3+}{ }_{1-\delta-\gamma} \mathrm{Sb}^{5+} \gamma\right)\left[\mathrm{Ni}^{2+}{ }_{1+2 \mathrm{x}-\delta} \mathrm{Fe}^{3+}{ }_{1+\delta+\gamma-3 \mathrm{x}} \mathrm{Sb}^{5+}{ }_{\mathrm{x}-\gamma}\right] \mathrm{O}^{2-}{ }_{4}
$$

This cation distribution was deduced from values of the magnetic moments and intensities of X-ray diffraction data assuming a collinear Néel spin structure. A formula, which describes the cationic distribution of the specific composition $\mathrm{FeNi}_{5 / 3} \mathrm{Sb}_{1 / 3} \mathrm{O}_{4}$ provided the antimony content is confined to the B-site, is given by

$$
\left(\mathrm{Ni}^{2+}{ }_{\mathrm{y}} \mathrm{Fe}^{3+}{ }_{1-\mathrm{y}}\right)\left[\mathrm{Ni}^{2+}{ }_{5 / 3-\mathrm{y}} \mathrm{Fe}^{3+}{ }_{\mathrm{y}} \mathrm{Sb}^{5+}{ }_{1 / 3}\right] \mathrm{O}_{4}
$$

Blasse reported an amount of $\mathrm{Ni}^{2+}$ in tetrahedral site of $\mathrm{y}=0.29[12,13]$; in contrast, Evans [14] found y-values up to 0.68 on compounds of the same composition. Later, unique ${ }^{57} \mathrm{Fe}$, ${ }^{61} \mathrm{Ni}$ and ${ }^{121} \mathrm{Sb}$ Mössbauer studies of the spinel ferrite $\mathrm{FeNi}_{5 / 3} \mathrm{Sb}_{1 / 3} \mathrm{O}_{4}$ [15] confirmed that the $\mathrm{Sb}^{5+}$ cations are located only in octahedral sites and indicated an y-value of 0.70 , and in fact a non-collinear spin structure.

X-ray (XRPD) or neutron powder diffraction (NPD) can be used to determine the cation distribution, provided that the coherent scattering lengths differ significantly for the different ions. However, in the case of NFO spinels, the X-ray scattering factors for Ni and Fe are very close, making it difficult to obtain accurate values of the cation occupancies from XRPD data. The situation is somewhat improved using NPD, but, unfortunately, the difference in the coherent scattering lengths of $\mathrm{Fe}$ and $\mathrm{Ni}$ for neutron scattering is only slightly greater than those in X-ray scattering $\left(0.95 \times 10^{-12}\right.$ and $1.00 \times 10^{-12} \mathrm{~cm}$, and $1.12 \times 10^{-12}$ and $1.14 \times 10^{-12} \mathrm{~cm}$ respectively). On the other hand, the NPD magnetic scattering amplitudes for $\mathrm{Fe}^{3+}$ and $\mathrm{Ni}^{2+}$ are $1.35 \times 10^{-12}$ and $0.54 \times 10^{-12} \mathrm{~cm}$, respectively, allowing accurate determination of arrangement and values of the magnetic moments.

In the present article, we have combined XRPD and NPD techniques with Mössbauer and Xray absorption spectroscopy and SQUID magnetometry in order to study the structural and magnetic properties of two spinels with chemical formula close to $\mathrm{Fe}_{0.8} \mathrm{Ni}_{1.8} \mathrm{Sb}_{0.4} \mathrm{O}_{4}$. The 
actual chemical composition as well as ionic states and site-occupancy of the $\mathrm{Ni}^{2+}, \mathrm{Fe}^{3+}$, and

$\mathrm{Sb}^{5+}$ ions were determined and are discussed in the light of structural considerations, magnetic properties and earlier references.

\section{Experimental}

\subsection{Sample preparation}

To prepare the compound we used a traditional in air multiple-step solid state reaction method using the starting materials $\mathrm{NiCO}_{3}, \mathrm{Sb}_{2} \mathrm{O}_{4.35}$ and $\mathrm{Fe}_{2} \mathrm{O}_{3}$. The reactive powders were mechanically ground and mixed in an agate mortar in appropriate stoichiometric ratios. The mixtures were first calcinated at $700{ }^{\circ} \mathrm{C}$ for $8 \mathrm{~h}$ and then slowly cooled to room temperature (RT). After grinding they were annealed for $8 \mathrm{~h}$ at $900{ }^{\circ} \mathrm{C}$ and again slowly cooled to RT. The grinding and annealing was repeated several times increasing the temperature by steps of 100 ${ }^{\circ} \mathrm{C}$ up to $1300{ }^{\circ} \mathrm{C}$. In the final stage the mixture was heated to $1340{ }^{\circ} \mathrm{C}$ with a slope of 10 ${ }^{\circ} \mathrm{C} / \mathrm{min}$, annealed for $6 \mathrm{~h}$ and slowly cooled to room temperature. The resulting compound (around $0.5 \mathrm{~g}$ ) was prepared for XRPD and magnetic measurements (sample 1). In order to allow NPD measurements, a second batch of sample (sample 2) of mass $5 \mathrm{~g}$ was prepared using the same technology as was used to synthesize sample 1. The only difference between the two sample preparations was related to the size of the ceramic disks (two times larger diameter in the case of sample 2) and duration of the final annealing at $1340{ }^{\circ} \mathrm{C}$ ( 4 and 6 hours, respectively). It was found from XRPD that the products of synthesis under these conditions were a Ni-Fe-Sb-O spinel (NFSO). Sample 2 contained a minute impurity phase of the binary oxide $\mathrm{NiSb}_{2} \mathrm{O}_{6}$, whereas sample 1 was found to be a pure, single phase spinel without impurity phases. One original motivation for this study was to prepare and study the $\mathrm{Ni}$ based synthetic analog of the natural mineral melanostibite, $\mathrm{Mn}_{2} \mathrm{FeSbO}_{6}$, which crystallizes in the structural type ilmenite [16]. However, the ilmenite $\mathrm{Ni}_{2} \mathrm{FeSbO}_{6}$ did not form in this preparation method.

\subsection{Chemical composition}

The chemical composition of the prepared ceramic samples was analyzed by energy dispersive spectroscopy (EDS) using a JEOL 840A scanning electron microscope and INCA 
4.07 (Oxford Instruments) software. The analyses were performed on 10 different grains in each of sample 1 and 2.

\subsection{X-ray diffraction}

The phase identification and purity of the powder samples were checked from X-ray powder diffraction (XRPD) patterns obtained with a D-5000 diffractometer using $\mathrm{Cu} \mathrm{K}_{\alpha}$ radiation. The ceramic sample of NFSO were crushed into powder in an agate mortar and suspended in ethanol. A Si substrate was covered with several drops of the resulting suspension, leaving randomly oriented crystallites after drying. The XRPD data for the Rietveld analysis were collected at room temperature on a Bruker D8 Advance diffractometer (Vantec positionsensitive detector, Ge-monochromatized $\mathrm{Cu} \mathrm{K}_{\alpha}$ radiation, Bragg-Brentano geometry, DIFFRACT plus software) in the $2 \theta$ range $10-152^{\circ}$ with a step size of $0.02^{\circ}$ (counting time was $15-20 \mathrm{~s}$ per step). The slit system was selected to ensure that the $\mathrm{X}$-ray beam was completely within the sample for all $2 \theta$ angles.

\subsection{X-ray absorption spectroscopy}

Extended X-Ray Absorption Fine Structure (EXAFS) data were collected in transmission mode around Ni K-edge at BM20 at Advanced photon source at Argonne National lab. A toroidal mirror is used to focus the X-ray beam at an energy cut-off of $32 \mathrm{keV}$. A KB mirror is then used to reduce the beam size down to $5 \square$ icrons in order to perform microprobe EXAFS measurements. Polycapillary optics were used to provide a high X-ray beam flux.

The two powder samples (samples 1 and 2) were finely milled and then shaped into two pellets of 20 micron thickness. The two pellets were then placed on a sample holder and aligned to the X-ray beam. The EXAFS data was processed and analyzed using Athena and Artemis software [17]. The spectra were background-subtracted, normalized, $\mathrm{k}^{3}$-weighted and Fourier transformed.

\subsection{Differential scanning calorimetry}

Heat capacities in the temperature range $200-800 \mathrm{~K}$ were obtained with help of a differential scanning calorimetry technique by using a DSCQ1000 from TA Instruments with the ceramic NFSO samples sealed in aluminum pans. The heating rate was maintained at $2{ }^{\circ} \mathrm{C} \min ^{-1}$. For determination of heat capacity, NIST synthetic sapphire (SRM 720) in the powder form was used as reference material. 


\subsection{Magnetic measurements}

The magnetization $\mathrm{M}$ was recorded as function of temperature in zero-field-cooled and fieldcooled conditions under various magnetic fields, and as function of magnetic field $\mathrm{H}$ at constant temperatures. The experiments were performed using a MPMSXL SQUID magnetometer and a PPMS6000 measurement system with VSM option, both from Quantum Design Inc.

\subsection{Mössbauer measurements}

The used computerized transmission Mössbauer spectrometer was of constant acceleration type, using 1024 cells for storing the unfolded data. The source, ${ }^{57} \mathrm{CoRh}$, was always held at room temperature. The absorbing material, typically around $10-15 \mathrm{mg} / \mathrm{cm}^{2}$, was crushed and mixed with a suitable amount $\left(\approx 50 \mathrm{mg} / \mathrm{cm}^{2}\right)$ of BN and spread evenly over the absorber disc, diameter $13 \mathrm{~mm}$. The amount of stuff was chosen to give optimal thicknesses, resulting in maximum of signal-to-noise ratio [18]. Low temperature measurements, down to $80 \mathrm{~K}$ were done using a flow cryostat of Oxford design, using nitrogen as cooling liquid. The folded spectra (512 cells) covering a velocity span of $\pm 15 \mathrm{~mm} / \mathrm{s}$ or less, have been least-square fitted using the "Recoil" program [19]. The center shift CS, being the sum of the true isomer shift and the second order Doppler shift, is given relative to metallic iron at room temperature. In the paramagnetic case, the magnitude of the quadrupole splitting, $|\mathrm{QS}|$, is given as the peak separation in the symmetrical (no texture or Goldanskii-Karyagin effects were assumed) doublet. The quadrupole shift $\varepsilon$, in the used dominant magnetic interaction model is defined as: $\left.=\left(\begin{array}{ll}\left(v_{6}\right. & v_{5}\end{array}\right)\left(\begin{array}{ll}v_{2} & v_{1}\end{array}\right)\right) / 4$. Here $v_{1}-v_{6}$ are the Lorentzian peak positions with increasing velocity in the fitted sextet. In this case $\varepsilon$ is given by: $=\frac{e Q V_{z z}}{4} \times \frac{3 \cos ^{2} 1+\sin ^{2} \cos 2}{2}$ Furthermore, the quadrupole splitting is given by: $Q S=\frac{e Q V_{z z}}{2} \times \sqrt{1+{ }^{2} / 3}$.

In these expressions the symbols have their conventional meanings. Especially, $\theta$ and $\phi$ are the polar and azimuthal angles for the magnetic field $\mathrm{H}$ in the principal axes system of the electric field gradient at the Mössbauer atom position. Moreover, the thin absorber approximation has been used, and thus the intensities for the peaks for increasing velocities in a sextet are proportional to $3: 2: 1: 1: 2: 3$. The hyperfine magnetic field, $\mathrm{H}$, is given in tesla. The 
intensity (given in \%) is the area of a sextet (or doublet for a non-magnetic pattern) below the base-line compared to the total absorbed area for the Mössbauer pattern.

\subsection{NPD measurements}

The neutron diffraction experiments were carried out at different temperatures at the LLB in Saclay, France. Two types of diffractometers installed on the guide lines of the Orpheae reactor were used. One is a two-axis spectrometer G4.1, equipped with a pyrolythic graphite monochromator which was tuned for the wavelength of $2.426 \AA$ and a $\mathrm{BF}_{3}$ linear multidetector, composed of 800 cells, which covers an $80^{\circ}(2 \theta)$ angular range. Depending on the sample studied, 10 to 12 measurement hours were necessary to record a spectrum with good statistics. NPD data were collected at low temperatures using a standard He cryostat and registered at different temperatures between 1.7 and $370 \mathrm{~K}$. The other diffractometer is a multicounter high-resolution diffractometer, 3T.2, composed of ten detectors with an angle space of $6^{\circ}$ and covering a $6 \pm 110^{\circ} 2 \theta$ range. A Ge single crystal was used as the monochromator and the wavelength was $1.2272 \AA$. 12 hours were necessary to record a spectrum with good statistics. In both cases, the powder sample studied was ground by hand with a pestle and an agate mortar, and loaded in a vanadium tube, approximately $5 \mathrm{~cm}$ long and with a diameter of $1 \mathrm{~cm}$.

Nuclear and magnetic refinements were performed by the Rietveld method, using the FULLPROF software [20]. The diffraction peaks were described by a pseudo-Voigt profile function, with a Lorentzian contribution to the Gaussian peak shape. A peak asymmetry correction was made for angles below $35^{\circ}$ in $2 \theta$. Background intensities were estimated by interpolating between up to 40 selected points (low temperature NPD experimental data) or described by a polynomial with six coefficients. The structure of cubic spinel $\mathrm{NiFe}_{2} \mathrm{O}_{4}$ (space group Fd-3m, standard setting) was taken as a starting model for the Rietveld refinements. The magnetic propagation vector was determined from the peak positions of the magnetic diffraction lines using the K-search software which is included in the FULLPROF refinement package [20]. Magnetic symmetry analysis was then done using the program BASIREPS [21]. The different allowed models for the magnetic structure were one by one tested against the measured data. Each structural model was refined to convergence, with the best result selected on the basis of agreement factors and stability of the refinement. 


\section{$\underline{\text { Results }}$}

\section{Chemical and structural characterizations}

Representative SEM micrographs of sample 1 and sample 2 of the prepared spinel are shown in figure 1. It is seen that both samples consist of a uniform distribution of crystallites, which are slightly larger for sample 1 .

The EDS analyses were performed on 10 crystallites for each sample. In sample 2, a few crystallites contained no iron, these could be assigned to belong to an impurity phase detected in the Rietveld refinement process: the corresponding set of very small peaks was indexed as $\mathrm{NiSb}_{2} \mathrm{O}_{6}$ (s.g. P4 $/$ mnm, $a=4.641(2) \AA$ and c=9.219(3) $\AA$ [21]; mass percentage content $<4 \%$ ). The tested main phase crystallites revealed good cationic homogeneity for both samples, and their $\mathrm{Fe}: \mathrm{Ni}: \mathrm{Sb}$ ratio leads to the approximate cationic formula:

$$
\begin{aligned}
& \mathrm{Fe}_{0.87(2)}: \mathrm{Ni}_{1.74(2)}: \mathrm{Sb}_{0.39(2)}(\text { Sample1) } \\
& \mathrm{Fe}_{0.74(2)}: \mathrm{Ni}_{1.84(2)}: \mathrm{Sb}_{0.42(2)}(\text { Sample } 2)
\end{aligned}
$$

The distribution of $\mathrm{Ni}^{2+}$ and $\mathrm{Fe}^{3+}$ cations among the tetrahedral and octahedral sites of the two samples was first studied using XRPD data sets (see Figure 2). Taking into account these formula, the spinel phase was very well refined with the cubic $\mathrm{F} d-3 m$ space group and the usual atomic positions of the spinel structure, i.e., tetrahedral site: $(8 a: 1 / 8,1 / 8,1 / 8)$; octahedral site: $(16 d: 1 / 2,1 / 2,1 / 2)$ and $\mathrm{O}(32 \boldsymbol{e}: \boldsymbol{u}, \boldsymbol{u}, \boldsymbol{u})$. (see Figure 3). The present system consists of three different types of cations, which are distributed over the tetrahedral and octahedral sites. In the data refinement process, the cation occupancies were varied carefully because the coherent scattering lengths of $\mathrm{Ni}$ and Fe cations only slightly differ. Additional sets of constrains were used in the Rietveld refinements: The cationic ratios obtained from the above EDS analysis, and the ratio between Fe cations distributed between tetrahedral and octahedral sites (obtained from Mössbauer data, see below). Sb is generally known to occupy the $16 \mathrm{~d}$ site [12-15, 23-27]. Therefore, this cation was fixed on the octahedral site and the occupancies of $\mathrm{Ni}$ and $\mathrm{Fe}$ were varied over the two sites within the stoichiometric constraints and the Mössbauer data. The results of the refinements of cation occupancy at different sites establish the following cation distributions:

$$
\begin{aligned}
& \left(N i_{0.26(2)} F e_{0.74(2)}\right)\left[N i_{1.48(2)} F e_{0.13(2)} S b_{0.39(2)}\right] \text { ( Sample 1) } \\
& \left(N i_{0.40(2)} F e_{0.60(2))}\left[N i_{1.44(2)} F e_{0.14(2)} S b_{0.42(2)}\right]\right. \text { ( Sample 2) }
\end{aligned}
$$


In which parentheses and square brackets denote tetrahedral and octahedral sites, respectively. The minimum difference pattern plots in Fig. 2 confirm the goodness of the fits. The values of unit cell size $\boldsymbol{a}$, and oxygen coordinate $\boldsymbol{u}$ varied for sample 1 and sample 2 (see Table 1). Also, the degree of inversion (defined by the fraction of the Ni sites occupied by Fe cations) is not the same for these two samples.

The ionic radii of octahedral $\mathrm{Ni}^{2+}{ }_{\mathrm{B}}$ and $\mathrm{Fe}^{3+}{ }_{\mathrm{B}}$ are 0.690 and $0.645 \AA$, respectively, and those of $\mathrm{Ni}^{2+}{ }_{\mathrm{A}}$ and $\mathrm{Fe}^{3+}{ }_{\mathrm{A}}$ are 0.550 and $0.490 \AA$, respectively [27]. In a rough approximation, a large (resp. low) value of $\boldsymbol{a}$ is related to more (resp. fewer) $\mathrm{Ni}^{2+}$ cations occupying tetragonal sites. Thus sample $1(\mathrm{a}=8.4163 \AA)$ is expected to contain fewer $\mathrm{Ni}^{2+}{ }_{\mathrm{A}}$ than sample $2(\mathrm{a}=8.4187 \AA)$. As $\mathrm{Ni}^{2+}$ is less stable in tetrahedral than in octahedral site, one may assume that the higher the amount of $\mathrm{Ni}^{2+}{ }_{\mathrm{A}}$ species, the lower the temperature of reduction of the spinel. An attempt to refine the main phase with the composition and site distribution proposed by Blasse for $\mathrm{FeNi}_{5 / 3} \mathrm{Sb}_{1 / 3} \mathrm{O}_{4}[12,13]$ was made; however, this led to significantly worse results.

\section{Mössbauer and EXAFS measurements}

We have obtained information on local environment and ionic states of $\mathrm{Fe}$ and $\mathrm{Ni}$ cations using Mössbauer and EXAFS spectroscopy respectively.

Mössbauer spectra were recorded at both room temperature and at $80 \mathrm{~K}$. As seen in Figure 4 for sample 2 , the room temperature spectrum is characterized by 6 broad profiles, broader when further away from the center of the spectrum. The first and sixth profile (numbered with increasing velocity) show shoulders indicating two groups of hyperfine fields. Each group was fitted using a Voight-profile distribution of hyperfine fields [29]. The two groups were characterized by different CS-values, average hyperfine fields and intensities; see Table 2 and Figure 4. Besides the two major sextets in the spectrum there is also a weak singlet in the centre ( $\approx 2 \%$ in intensity) assigned to an unknown impurity phase, not included in the discussions. The same Mössbauer absorber was also measured at 80(2) K where the distributions of hyperfine fields are much smaller. As a result, it was possible to fit the spectrum only using two Lorentzian sextets where the $(1,6)$ - and $(2,5)$-peaks in the sextet were allowed to be broader than the (3,4)-peaks, see Table 2 and Figure 4 . The assignments of Mössbauer sextets (and doublets) to the two crystallographic sites are straightforward. The measured center shifts are characteristic of high-spin trivalent iron in octahedral coordination and in tetrahedral coordination. The two lines are in the ratio $\mathrm{Fe}^{3+}{ }_{\mathrm{B}} / \mathrm{Fe}^{3+}{ }_{\mathrm{A}}=15 / 85$. In spinels, 
being in the magnetic state, the octahedral (B) site normally gives higher hyperfine fields as well as center shifts [30] than the tetrahedral site (A). $\mathrm{CS}(\mathrm{B})-\mathrm{CS}(\mathrm{A}) \approx 0.10 \mathrm{~mm} / \mathrm{s}$ in quite many oxy-spinels [31], as also achieved by us here. The obtained cation distribution with $\approx 18$ $\%$ of iron residing at the octahedral site is in rough agreement with other reports. Blasse $[12,13]$ found all $\mathrm{Sb}$ to reside at the octahedral site and about $20 \%$ of Ni to be at the tetrahedral site. M. K. El-Nimr et al. proposed twice as much iron at tetrahedral site compared to at octahedral site, a deviation from our results, but showing the same tendency [23]. Similar results were obtained for sample 1 and mineral filipstadite [33-35] with spinel structure having in its composition $\mathrm{Sb}^{5+}$. The Mössbauer spectrum for sample 1 is rather similar to the one obtained for sample 2 . As the main conclusion it was established that in our spinel samples iron prefers residing at the tetrahedral site.

Information on the local environment of Ni cations was instead obtained from EXAFS spectra, which were found similar for both samples 1 and 2. As seen in Figure 5, two dominant peaks may be observed in the Fourier transform (FT) of the spectra around $1.63 \AA$ and $2.58 \AA$. Here we show the Fourier transform of the experimental data directly, without any phase correction. These peaks can be associated with single scattering contributions to the EXAFS from the oxygen $(1.63 \AA)$ and metal $(2.58 \AA)$ atoms, given the form of the associated scattering amplitudes in reciprocal space. These peaks were fitted considering both single and double peak fits, yielding similar values within the error. The first peak, characteristic of oxygen backscattering, gives a distance of 2.00(3) A. The second peak, characteristic of metal backscattering, yields a distance of 2.90(3). Both peaks indicate an equal coordination, close to 6, which may be compatible with the B-O and B-B distances presented in Table 1, with Ni cations mainly on octahedral B sites. The Debye-Waller factors are non negligible of order 0.007(3) A indicating some disorder in the structure.

\section{Magnetic behavior}

The magnetic properties of samples 1 and 2 were investigated by means of magnetization $M$ measurements as function of temperature and magnetic fields $H$. The upper panel of Figure 6 shows $\mathrm{M}(\mathrm{T}) / \mathrm{H}$ recorded in $20 \mathrm{Oe}$ (sample 1) and 50 Oe (sample 2) in zero-field-cooled (ZFC) and field-cooled (FC) protocols. As seen in the figure both samples show a similar behavior, reminiscent of that of ferrimagnets with compensation points [36-40]. Depending on the sign of the magnetization attained during the initial cooling in nominally zero (but in reality finite background magnetic field), the ZFC magnetization is first decreasing or increasing with 
increasing temperature. It then increases significantly from above $150 \mathrm{~K}$, up to about $305 \mathrm{~K}$.

Above that temperature the magnetization continuously decreases. The FC magnetization recorded on cooling subsequent to the ZFC measurement, initially closely follows the ZFC one at high temperatures. At about $340 \mathrm{~K}$, The FC curve starts to deviate from the ZFC one, but the overall behavior in the $300-150 \mathrm{~K}$ temperature region is similar to that of the ZFC curve, and the FC magnetization decreases as the temperature decreases. For both samples 1 and 2, the FC curve crosses the ZFC curve near $140 \mathrm{~K}$, and remains lower than the ZFC curve down to the lowest temperature. $\mathrm{ZFC} / \mathrm{FC}$ curves recorded from room temperature to $900 \mathrm{~K}$ in an applied field of $1 \mathrm{kOe}$ are depicted in the inset. An inflection can be observed in the ZFC magnetization below $650 \mathrm{~K}$, associated with some irreversibility, which may suggest onset of magnetic order. At lower temperatures the magnetization increases, somewhat more sharply below $480 \mathrm{~K}$.

The temperature dependent $\mathrm{ZFC} / \mathrm{FC}$ magnetization recorded in larger magnetic fields, and magnetization recorded as a function of magnetic field, were found to be qualitatively similar for samples 1 and 2; in the following only data obtained from sample 2 is shown. ZFC/FC measurements recorded in larger magnetic fields (from $1 \mathrm{kOe}$ to $90 \mathrm{kOe}$ ) are plotted in the lower panel of Figure 6. As seen in the figure, the irreversibility between the ZFC/FC curves is shifted down to about $300 \mathrm{~K}$ in $1 \mathrm{kOe}$, and to about $200 \mathrm{~K}$ in $5 \mathrm{kOe}$. In $20 \mathrm{kOe}$, the ZFC/FC data are quite similar to one another, and nearly identical for larger fields. As the irreversibility vanishes, the crossing point between ZFC and FC curves near 140K is replaced by a minimum in the magnetization curves. The maximum in the FC magnetization, observed near $280 \mathrm{~K}$, seems not to be dependent on the magnitude of the magnetic field.

Hysteresis loops recorded at different temperatures are shown in the upper panel of Figure 7. The curves suggest a weakly ferromagnetic or ferrimagnetic behavior, with a magnetization which increases quasi linearly above $30 \mathrm{kOe}$ at all temperatures. At low temperatures, the coercivity of the sample is relatively large, about $6 \mathrm{kOe}$ at $\mathrm{T}=2 \mathrm{~K}$. The shape of the $\mathrm{M}(\mathrm{H})$ curve is preserved as the temperature increases up to $150 \mathrm{~K}$ (near the compensation-like point in $\mathrm{ZFC} / \mathrm{FC}$ curves) while the coercivity decreases. Above $150 \mathrm{~K}$, the shape of the $\mathrm{M}(\mathrm{H})$ curves becomes less and less inclined as the temperature increases (see e.g. curves obtained at 200 and $300 \mathrm{~K}$ in the upper panel of Figure 7) but the overall magnetization increases. Above 300 $\mathrm{K}$ the magnetization decreases again as the temperature increases further, in agreement with the high-field magnetization data shown in the lower panel of Figure 6. An exchange bias phenomenon is often observed in ferrimagnetic compounds[36-40]. We have collected $\mathrm{M}(\mathrm{H})$ 
curves recorded at temperatures above and below the compensation point after field cooling (not shown), however no horizontal shift of the hysteresis curves was observed.

Considering the chemical formula $\mathrm{Fe}_{0.87} \mathrm{Ni}_{1.74} \mathrm{Sb}_{0.39} \mathrm{O}_{4}$ obtained for Sample 1, with molecular weight of $262.16 \mathrm{~g} / \mathrm{mol}, 1 \mathrm{emu} / \mathrm{g}$ in Figure 6 and 7 amounts to about $0.047 \mu_{\mathrm{B}} / \mathrm{f} . \mathrm{u}$. By extrapolating the quasi-linear high-field behavior of $\mathrm{M}(\mathrm{H})$ to zero magnetic field, we can obtain an estimate of the spontaneous moment associated with the hysteresis loops. This moment is plotted in the lower panel of Figure 7 as a function of temperature. The magnitude of the moment is rather small and seems to confirm the expected antiferromagnetic coupling of the A and B sublattices. This is confirmed by the NPD results yielding antiferromagnetic alignment of the $A$ and $B$ lattice moments and a small net moment $\mathrm{M}_{B}-\mathrm{M}_{\mathrm{A}}$ that varies with temperature in agreement with $\mathrm{M}(\mathrm{T})$ from the magnetization measurements. An observation supported by both the magnetization measurements and the NPD is that this rare earth free ferrimagnet apparently exhibits a compensation point at about $200 \mathrm{~K}$.

\section{Heat capacity}

On the zero-field heat capacity curve $\mathrm{C}_{\mathrm{p}}(\mathrm{T})$ obtained for both samples (see Figure 8 ), some anomalies emerge. The temperature of a clear maximum can be determined with an experimental uncertainty lower than $5 \mathrm{~K}$ and occurs at $630 \mathrm{~K}$ and $605 \mathrm{~K}$ for sample 1 and sample 2, respectively. Comparing this calorimetric data with the magnetic results of the previous section and the structural characterizations reported in $[41,42]$ this maximum is related to magnetic ordering, i.e. a ferrimagnetic phase transition, $T_{N}$. This temperature has been reported to strongly depend on the inversion parameter $\gamma$ [37]. Different authors have published slightly different values of $\gamma$ and $T_{N}$ with a difference in $T_{N}$ as high as $30 \mathrm{~K}$.

\section{Magnetic Contribution to the Neutron-Diffraction Pattern}

The low angle reflections in the NPD patterns increase significantly at lower temperatures (see Figures 9, 10). These added contributions are of magnetic origin. For unpolarized neutrons diffracted from a ferromagnetic or ferrimagnetic sample, there is no coherence between the nuclear and magnetic diffraction. The total scattering intensity is therefore the sum of the nuclear and magnetic diffraction intensities. In most of the oxy-spinels with paramagnetic ions at the tetrahedral and octahedral sites, the crystallographic and magnetic unit cells are the same, having identical symmetry relations $[43,44]$, therefore low angle Bragg peaks contain magnetic contributions. 
To analyze the diffraction patterns, we used a two-phase profile refinement method: one phase involves the nuclear scattering contribution, while the second involves the magnetic scattering contribution. Compared with the corresponding NPD pattern at $600 \mathrm{~K}$ the pattern at $295 \mathrm{~K}$ and $2 \mathrm{~K}$ showed the expected overall increase in intensity, but no separate magnetic peaks were observed (Figure 9). The magnetic unit cell below $\mathrm{T}_{\mathrm{N}}$ thus appears to coincide with the chemical unit cell. Assuming the spin moments at $2 \mathrm{~K}$ to be antiparallel and completely collinear, the net magnetization would be $0.15(6) \mu_{\mathrm{B}} / \mathrm{f}$.u. Any long range canting angle would result in the existence of the (200) magnetic Bragg peak. No intensity is observed in theoretical position of (200) reflection either at 295 or $2 \mathrm{~K}$.

Rietveld analysis was carried out using the FULLPROF suite to refine a minimal set of variables, i.e., the lattice parameter, the internal oxygen coordinate $\mathbf{u}$, and the magnetic moments of the tetrahedral and octahedral sites, as well as background, and profile coefficients (Table 1). Figure 11 shows the corresponding observed and calculated diffraction patterns at 650, 295 and $2 \mathrm{~K}$. The inverse spinel structure refined from our NPD data agrees well with that determined by XRD. In this crystal structure, it is supposed that $\mathrm{Fe}^{3+}$ and $\mathrm{Ni}^{2+}$ occupies the tetrahedral sites at $(1 / 8,1 / 8,1 / 8)$ and $\mathrm{Ni}^{2+}, \mathrm{Fe}^{3+}$ and $\mathrm{Sb}^{5+}$ occupy the octahedral sites at $(1 / 2,1 / 2,1 / 2)$ with oxygen atoms at $(\boldsymbol{u}, \boldsymbol{u}, \boldsymbol{u})$ positions (Figure 3$)$. The values of $\boldsymbol{u}$ from the refinements are found to be independent of temperature: two data sets from different diffractometers yield a very similar value $\boldsymbol{u}$. The lattice parameter $\boldsymbol{a}$ smoothly increases with temperature (see Figure 12).

A small systematic difference between the $\boldsymbol{a}$ values refined from two NPD data sets is probably due to the uncertainty in the wavelength calibrations. All possible configurations where magnetic orders are represented by parallel or antiparallel magnetic moments between the cations were tested. The magnetic refinements confirm a collinear ferrimagnetic structure (magnetic space group F-1). In spinel oxides, the magnetic moments are mainly from the parallel uncompensated electron spins of the individual ions and according to Néel's two sublattice model the spin alignments in the two sublattices are antiparallel. The net magnetic moment per formula unit is expressed as: $M_{n e t}=M_{\mathrm{B}}-M_{\mathrm{A}}$, where $M_{\mathrm{A}}$ and $M_{\mathrm{B}}$ are tetrahedral and octahedral sublattice magnetic moments, respectively. The sublatttice magnetic moments $M_{\mathrm{A}}$ and $M_{\mathrm{B}}$ have been derived from the refinement of the magnetic moments of the individual cations and their occupation on the different sites. $M_{n e t}$ has been determined from the difference of the two sublattice moments. The upper panel of Figure 13 shows the variation of $M_{\mathrm{A}}, M_{\mathrm{B}}$ and $M_{n e t}$ as well as the variation of the $\mathrm{Fe}^{3+}$ and $\mathrm{Ni}^{2+}$ moments on the different sites as a function of temperature. The sublattice moments as well as the moment of the individual 
cations show a regular continuous decrease with increasing temperature. The lower panel of Figure 13 shows the temperature dependence of the net magnetic moment, which shows a weak net moment that varies with temperature quite similarly as the measured spontaneous magnetization shown in the lower panel of Figure 7.

The agreement between the refinements of two data sets is quite good, although there is a systematic difference of about $0.2 \mu_{\mathrm{B}}$ between the values for the tetrahedral site moments. The origin of this discrepancy is not clear, but it may be related to strong correlations with the thermal parameters in the fits.

In the spinel structure (following [45]), the oxygen coordinate $\boldsymbol{u}$ is related to the $\boldsymbol{A}$ (or $\boldsymbol{B})-\boldsymbol{O}$ bond lengths $\mathrm{d}_{\mathrm{A}}$ and $\mathrm{d}_{\mathrm{B}}$ at the $\boldsymbol{A}$ and $\boldsymbol{B}$ sites by

$$
\boldsymbol{d}_{\boldsymbol{A}}=\sqrt{ } 3 a(\boldsymbol{u}-1 / 8) \text { and } \boldsymbol{d}_{\boldsymbol{B}}=\boldsymbol{a}(1 / 2-\boldsymbol{u}) .
$$

From the fit parameters, we find for sample $2 \boldsymbol{d}_{\boldsymbol{A}}=1.93(1) \AA$ and $\boldsymbol{d}_{\boldsymbol{B}}=2.03(1) \AA$. These values are in a good agreement with those for the $\boldsymbol{A}-\boldsymbol{O}$ and $\boldsymbol{B}-\boldsymbol{O}$ bond lengths calculated from ionic radii $\left(\boldsymbol{d}_{\boldsymbol{A}}=1.90 \AA\right.$ and $\left.\boldsymbol{d}_{\boldsymbol{B}}=2.05 \AA\right)$. The small difference may indicate a greater covalent character of bonds.

\section{Discussion}

The XRD lattice parameter is larger for sample 2 than for sample 1 - this finding concords with the derived sample composition and distribution on the A and B sites: sample 1 is somewhat more rich on $\mathrm{Sb}$ and consequently significantly more Ni rich, especially on the tetragonal position ( 0.40 (sample 2) compared to 0.26 (sample 1)). The larger ionic radius of $\mathrm{Ni}^{2+}$ compared to $\mathrm{Fe}^{3+}$ corroborates this result.

Also, the cation distribution in spinels containing transition metals can usually be correlated to crystal field stabilization energies and cation radii of the transition metal ions in octahedral and tetrahedral environments [46]. In our compounds there is a clear preference of Sb to occupy only the octahedral sites. According to [46,47], larger cations have preference for tetrahedral sites.

With our NPD experiments we are able to deduce what the elemental distribution among the sites is, but we are unable to univocally assign the oxidation state of Ni. The valence state of Fe was determined to be exclusively $\mathrm{Fe}^{3+}$ from the current Mössbauer data. 
The saturation magnetization of the collinear regime, referred to as $M$ sat(col.), can be approximated by a smooth extrapolation of the $M(T)$ curve down to lowest temperatures, leading to $\approx 0.05$ (5) $\mu_{\mathrm{B}} / \mathrm{f}$.u. This experimental value must be compared to the theoretical one, i.e., $\left|M_{\mathrm{A}}-M_{\mathrm{B}}\right|$, where $M_{\mathrm{A}}$ and $M_{\mathrm{B}}$ are the saturation magnetizations of the $\mathrm{A}$ and $\mathrm{B}$ sublattices, respectively.

In the spinel structure, the relation between lattice parameter and bond distances is expressed as [48]:

$$
a_{\text {calc }}=8 / 9\left(\sqrt{3} d_{\mathrm{A}-\mathrm{O}}+3 d_{\mathrm{B}-\mathrm{O}}\right)
$$

where $\boldsymbol{d}_{\boldsymbol{A}}$ and $\boldsymbol{d}_{\boldsymbol{B}}$ are the average bond lengths of the A and B site, respectively. Using the mean values of the ionic radii of the cations of our compounds, the so calculated lattice parameters are compared with those obtained from our Rietveld refinement of neutron data. The maximum deviation between the calculated and measured values is found to be less than $2 \%$.

\section{Concluding remarks}

Structural and magnetic properties of two spinel samples in the Fe-Ni-Sb-O system are reported. The Rietveld refinements of diffraction data used for determining the cation distribution among the tetrahedral (A) and octahedral (B) sites in the spinel-related structure and crystallographic characterization of the system indicate that the prepared samples possess a cubic spinel structure of Fd-3m symmetry between 1.7 and $650 \mathrm{~K}$. The refinement yielded a mixed cationic distribution with $\mathrm{Ni}^{2+}$ and $\mathrm{Fe}^{3+}$ being distributed over tetrahedral and octahedral sites in varying proportions while $\mathrm{Sb}$ was found to uniquely occupy the octahedral interstices. The magnetic structure below $\mathrm{T}_{\mathrm{N}}$ was determined to be Néel type where magnetic cations of $\mathrm{Ni}^{2+}$ and $\mathrm{Fe}^{3+}$ are in high spin state and the origin of the weak spontaneous magnetization is attributed to imperfect compensation of the A and B magnetic sublattices. It is concluded that the combination of neutron diffraction, Mössbauer and synchrotron measurements has given a clear picture of the cation distributions in spite of their being close in atomic numbers and scattering lengths. Of certain interest is that the investigated spinel system constitutes a rare case of a rare-earth free ferrimagnet with a compensation point 


\section{Acknowledgements}

We thank the Swedish Research Council (VR), the Göran Gustafsson Foundation, the Swedish Foundation for International Cooperation in Research and Higher Education (STINT), and the Russian Foundation for Basic Research for financial support. PNC/XSD facilities at the Advanced Photon Source, and research at these facilities, are supported by the US Department of Energy - Basic Energy Sciences, the Canadian Light Source and its funding partners, the University of Washington, and the Advanced Photon Source. Use of the Advanced Photon Source, an Office of Science User Facility operated for the U.S. Department of Energy (DOE) Office of Science by Argonne National Laboratory, was supported by the U.S. DOE under Contract No. DE-AC02-06CH11357. 
Table 1 Structural parameters, bond distances, bond angles at room temperature for NFSO spinels.

\begin{tabular}{|c|c|c|c|c|c|c|c|}
\hline \multicolumn{2}{|c|}{ Compound } & Sample1 & Sample2 & \multicolumn{4}{|c|}{ Sample2 NPD } \\
\hline \multicolumn{2}{|c|}{$\mathrm{T}, \mathrm{K}$} & 295 & 295 & 1.7 & 309 & 600 & 650 \\
\hline \multicolumn{2}{|c|}{ Lattice constant $(\AA)$} & $8.4163(1)$ & $8.4187(1)$ & $8.4067(1)$ & $8.4165(1)$ & $8.4413(2)$ & $8.4452(2)$ \\
\hline \multicolumn{2}{|c|}{ Oxygen position $\boldsymbol{u}$} & $0.2558(3)$ & $0.2547(3)$ & $0.2563(3)$ & $0.2568(3)$ & $0.2569(4)$ & $0.2568(4)$ \\
\hline \multirow{5}{*}{$\begin{array}{c}\text { Refine- } \\
\text { ment } \\
\text { quality } \\
\text { factors }\end{array}$} & $\mathrm{Rp}$ & 3.76 & 3.84 & 3.26 & 3.18 & 2.74 & 2.67 \\
\hline & Rwp & 5.65 & 5.73 & 5.31 & 5.09 & 3.61 & 3.43 \\
\hline & $\chi^{2}$ & 2.11 & 2.18 & 1.54 & 1.73 & 1.82 & 1.79 \\
\hline & $\mathrm{R}_{\mathrm{B}}$ & 5.13 & 5.21 & 2.12 & 2.29 & 4.03 & 4.08 \\
\hline & $\mathrm{R}_{\text {mag }}$ & - & - & 7.5 & 7.9 & - & - \\
\hline \multirow{5}{*}{$\begin{array}{l}\text { Bond } \\
\text { distance } \\
(\AA)\end{array}$} & $\mathrm{A}-\mathrm{O}$ & $1.902(1)$ & $1.891(1)$ & $1.912(1)$ & $1.922(1)$ & $1.928(2)$ & $1.929(2)$ \\
\hline & $\mathrm{B}-\mathrm{O}$ & $2.059(1)$ & $2.066(1)$ & $2.046(1)$ & $2.048(1)$ & $2.054(2)$ & $2.055(2)$ \\
\hline & $\mathrm{A}-\mathrm{A}$ & $3.644(1)$ & $3.645(1)$ & $3.640(1)$ & $3.645(1)$ & $3.655(2)$ & $3.657(2)$ \\
\hline & B-B & $2.976(1)$ & $2.977(1)$ & $2.972(1)$ & $2.976(1)$ & $2.984(2)$ & $2.986(2)$ \\
\hline & A-B & $3.489(1)$ & $3.490(1)$ & $3.485(1)$ & $3.489(1)$ & $3.5000(2)$ & $3.501(2)$ \\
\hline \multirow{6}{*}{$\begin{array}{l}\text { Bond } \\
\text { angle } \\
\left({ }^{\circ}\right)\end{array}$} & $\mathrm{O}-\mathrm{A}-\mathrm{O}$ & $109.5(1)$ & $109.5(1)$ & $109.5(1)$ & $109.5(1)$ & $109.5(1)$ & $109.5(1)$ \\
\hline & \multirow{3}{*}{$\mathrm{O}-\mathrm{B}-\mathrm{O}$} & 180 & 180 & 180 & 180 & 180 & 180 \\
\hline & & $87.4(1)$ & $87.8(1)$ & $87.1(1)$ & $86.7(1)$ & $86.8(1)$ & $86.8(1)$ \\
\hline & & $92.6(1)$ & $92.2(1)$ & $92.9(1)$ & 93.3(1) & 93.2(1) & 93.1(1) \\
\hline & A-O-B & $123.4(1)$ & $123.7(1)$ & $123.2(1)$ & $122.9(1)$ & $123.0(1)$ & $123.0(1)$ \\
\hline & B-O-B & $92.6(1)$ & $92.2(1)$ & $94.2(1)$ & 93.2(1) & 93.1(1) & 93.1(1) \\
\hline
\end{tabular}

Table 2 Fitting parameters of Mössbauer spectra at 295 and 80K for NFSO spinel.

\begin{tabular}{|c|c|c|c|}
\hline CS (mm/s) & H (Tesla) & AREA (\%) & Assignment \\
\hline \multicolumn{5}{|c|}{$295 \boldsymbol{K}$} \\
\hline $0.27(1)$ & $41.3(3)$ & $85(5)$ & tetrahedral \\
\hline $0.36(2)$ & $50.6(2)$ & $15(5)$ & octahedral \\
\hline \multicolumn{5}{|c|}{$80 \boldsymbol{K}$} \\
\hline $0.38(1)$ & $47.2(1)$ & $85(2)$ & tetrahedral \\
\hline $0.46(2)$ & $55.2(1))$ & $14(1)$ & octahedral \\
\hline
\end{tabular}




\section{Reference list}

[1] P.A. Cox in Transition Metal Oxides, Clarendon Press, Oxford, United Kingdom, 1992.

[2 ] T. Birol, N.A. Benedek, H. Das, A.L. Wysocki, A.T. Mulder, B. M. Abbett, E.H. Smith, Curr. Opin. Solid State Mater. Sci. 16, 227 (2012).

[3] N.A. Benedek, A.T. Mulder, C. J. Fennie, J. Solid State Chem. 195, 11 (2012).

[4] M. Sugimoto, J. Am. Ceram. Soc. 82 ,269 (1999).

[5 M.A. Willard, L.K. Kurihara, E.E. Carpenter, S. Calvin, V.G. Harris, Int. Mater. Rev. 49, 125 (2004).

[6] V. Sepelak, A. Feldhoff, P. Heitjans, F. Krumeich, D. Menzel, F.J. Litterst, I. Bergmann, K.D. Becker, Chem. Mater. 18, 3057 (2006).

[7] S.P. Crane, C. Bihler, M.S. Brandt, S.T.B. Goennenwein, M. Gajek, R.

Rameshhttp://www.sciencedirect.com/science/article/pii/S0304885308009311 - aff1 J. Magn. Magn. Mater. 321, L5 (2009).

[8] Q. Zhang, K. Singh, F. Guillou, C. Simon, Y. Breard, V. Caignaert, V. Hardy, Phys. Rev. B. 85, 054405 (2012).

[9] K.E.Sickafus, J.M.Wills, J. Amer. Ceram. Soc. 82, 3279 (1999).

[10] H. Müller-Buschbaum, J. Alloys Compds 349, 49 (2003).

[11] R. Hill, J. Craig and G. Gibbs, Phys. Chem. Miner. 4 ,317 (1979).

[12] G. Blasse, Philips Res. Rep. Suppl. No. 3 (1964).

[13] G. Blasse, J. Inorg. Nucl. Chem. 26, 1191 (1964).

[14] B.J. Evans, Mössbauer effect methodology 4,139 (1968).

[15] G.Dehe, P.Griesbach, P.Gutlich Appl. Phys. A 43, 213 (1987).

[16] R. Mathieu, S. A. Ivanov, I. V. Solovyev, G. V. Bazuev, P. Anil Kumar, P. Lazor, and P. Nordblad, Phys. Rev. B 87, 014408 (2013); P. B. Moore, Amer. Mineral. 53, 1104 (1968).

[17] B. Ravel and M. Newville, J. Synchrotron Radiat. 12, 537 (2005).

[18] D.G. Rancourt, A.M. McDonald, A.E. Lalonde, J.Y. Ping, Am. Mineral. 78, 1 (1993)

[19] K.Lagarec, D.G. Rancourt, Nucl. Instr. Meth. B 129, 266 (1997).

[20] J. Rodriguez-Carvajal, Physica B 192, 55 (1993).

[21] J. Rodriguez-Carvajal, BASIREPS: a program for calculating irreducible representations of space groups and basis functions for axial and polar vector properties, 2007. 
[22] H. Ehrenberg, G. Wltschek , J. Rodriguez-Carvajal , T..Vogt, J. Magn. Magn. Mater. 184, 111 (1998).

[23] M.K. El-Nimr, H.A. Saleh and M. K. Fayek Appl. Phys. A 38, 67 (1985).

[24] M.K. El Nimr, M.A. Ahmed, M.A. El Hiti, J. Mater. Sci. Lett. 13, 1500 (1994).

[25] K.P. Belov, A.N. Goryaga, R.R. Annaev, Soviet Physics - Solid State 33, 1407 (1991).

[26] G. Dehe, J. Suwalski, Hyperfine Interactions 28, 659 (1986).

[27] M.K. El-Nimr, H.A. Saleh, M.K. Fayek, Nucl. Sci. J. 22, 1 (1985).

[28] R. D. Shannon Acta Cryst. A32, 751(1976).

[29] D. G. Rancourt, J. Y. Ping, Nucl. Instr. Meth. B 58, 85 (1991)

[30] N.N. Greenwood, T. C. Gibb: Mössbauer Spectroscopy (Chapman and Hall, London 1971) pp. 259-268.

[31] J. J. van Loef, Physica 32, 2102(1966).

[32] G.A. Sawatsky, F. van der Woude ans A.H. Morrish, Phys. Rev. 183, 383 (1969).

[33] P.Bonazzi, L.Chelazzi, L.Bindi, Amer. Miner. 98, 361 (2013).

[34] P.J. Dunn, D.R. Peacor, A.J. Criddle, C.J. Stanley, Amer. Miner., 73, 413 (1988).

[35] Holtstam, D., Nysten, P., and Gatedal, K., Mineralogical Magazine, 62, 395(1998).

[36] J.C.G. Tedesco, M.J.M. Pires, A. Magnus G. Carvalho, V.S.R. de Sousa, L.P. Cardoso, and A.A. Coelho, J. Magn. Magn. Mater. 339, 6 (2013).

[37] K. Szymanski, B. Kalska, D. Satula, L. Dobrzynski, A. Broddefalk, R. Wappling, and P. Nordblad, J. Magn. Magn. Mater. 251, 271 (2002).

[38] H. G. Zhang, W. H. Wang, E. K. Liu, X. D. Tang, G. J. Li, J. L. Chen, H. W. Zhang, and G. H. Wu, Phys. Status Solidi B 250, 1287-1292 (2013).

[39] L.H. Yin, Y. Liu, S.G. Tan, B.C. Zhao, J.M. Dai, W.H. Song, and Y.P. Sun, Mater. Res. Bull. 48, 4016 (2013).

[40] R. Padam, S. Pandya, S. Ravi, A. K. Nigam, S. Ramakrishnan, A. K. Grover, and D. Pal, Appl. Phys. Lett. 102, 112412 (2013).

[41] A.A. El-Sharkawy, A.M. Abousehly, El-S.M. Higgy, High Temperatures - High Pressures 18, 265 (1986).

[42] A.A. El-Sharkawy, A.M. Abousehly, El-S.M. Higgy, Phys.Stat. Sol.(A) 95, 605 (1986).

[43] J.M.Hastings, L.M.Corless, Rev. Mod. Phys. 25, 114 (1953). 
[44] L.Mandrav Rao, Bull. Mater. Sci. 7, 303 (1985).

[45] L.Chang, B.D.Rainford, J. R. Stewart, C. Ritter, A. P. Roberts, Y. Tang, Q.Chen, J. Geophys. Res: Solid Earth 114, 1978 (2009).

[46] A. Navrotsky, O.J. Kleppa, J. Inorg. Nucl. Chem. 29, 2701(1967)

[47] H.SI.C. O'Neill, A. Navrotsky, Amer. Miner. 68, 181 (1983); ibidem 69, 733 (1984).

[48] S. Krupicka, P. Novak, in: E.P. Wohlfarth (Ed.), Ferromagnetic Materials, vol. 3, North-Holland, 1982. 


\section{Figure Captions}

Figure 1. Scanning electron microscopy image of sample 1 (a) and sample 2 (b).

Figure 2. Experimental (crosses), calculated, and difference (solid lines) XRPD pattern of two spinel samples at the end of refinement. The ticks mark the Bragg positions for the spinel phase (upper) and $\mathrm{NiSb}_{2} \mathrm{O}_{6}$ (lower).

Figure 3. Polyhedral representation of spinel crystal structure.

Figure 4. Fitted Mössbauer spectrum for spinel sample 2 recorded at 295K (a) and $78 \mathrm{~K}$ (b).

Figure 5. The magnitude of the Fourier Transform (FT) of the fine structure weighted by $\mathrm{k}^{3}$ $\left(\mathrm{k}^{3} \chi(\mathrm{k})\right)$ is shown (blue line) in real space, together with the result of a double peak fit (red line). The data as shown here are not phase corrected.

Figure 6. Upper panel: Temperature dependence of $\mathrm{M} / \mathrm{H}$ recorded in 20 Oe for sample 1 (open circles) and 50 Oe for sample 2 (filled circles). As indicated with the arrows, the zerofield-cooled (ZFC) magnetization was recorded first on heating up to $390 \mathrm{~K}$, and the fieldcooled (FC) magnetization was recorded on a subsequent cooling down. The inset shows the corresponding ZFC/FC curves recorded in a larger field (1 kOe) up to higher temperatures. Lower panel: Temperature dependence of the magnetization $\mathrm{M}$ recorded in $\mathrm{ZFC} / \mathrm{FC}$ conditions under different magnetic fields for sample 2.

Figure 7. Upper panel: Hysteresis loops - magnetization recorded as function of magnetic field as the field is swept from 0 to $+90 \mathrm{kOe},+90$ to $-90 \mathrm{kOe}$, and -90 to $+90 \mathrm{kOe}-$ at various temperatures for samples 2. Only results for selected temperatures and specific parts of the $\mathrm{M}(\mathrm{H})$ curves are shown, in order to show both low-field and high-field regimes with more clarity. Lower panel: Magnetic moment extracted from the $\mathrm{M}(\mathrm{H})$ curves depicted in the upper panel (see main text) at all measured temperatures. For some temperatures, the magnetic field was swept only up to $+/-50 \mathrm{kOe}$, yet yielding similar results. Typical errorbars of $10 \%$ are added as a guide for the eye.

Figure 8. Temperature dependence of the heat capacity for sample 1 and sample 2.

Figure 9. Evolution of the NPD patterns of sample 2 with temperature (data from G4.1 diffractometer).

Figure 10. Evolution of the NPD patterns of sample 2 with temperature (data from 3T2 diffractometer).

Figure 11. The observed, calculated, and difference NPD patterns of sample 2 after Rietveld refinement of the nuclear and magnetic structure at different temperatures: $650 \mathrm{~K}$ (a), $295 \mathrm{~K}$ (b) and $10 \mathrm{~K}(\mathrm{c})$. The ticks mark the nuclear and magnetic peaks for the spinel phase (upper (a); upper and middle (b,c)) and $\mathrm{NiSb}_{2} \mathrm{O}_{6}$ (lower).

Figure 12. Evolution of the lattice parameter of sample 2 with temperature.

Figure 13. Upper panel: Temperature dependence of the sublattice magnetization $\left(\mathrm{M}_{\mathrm{A}}\right.$ and $\mathrm{M}_{\mathrm{B}}$ ) and cation moments on the tetrahedral and octahedral sites. Lower panel: The net magnetic moment per formula unit $\left(\mathrm{M}=\mathrm{M}_{\mathrm{B}}-\mathrm{M}_{\mathrm{A}}\right)$ vs temperature for sample 2 . 


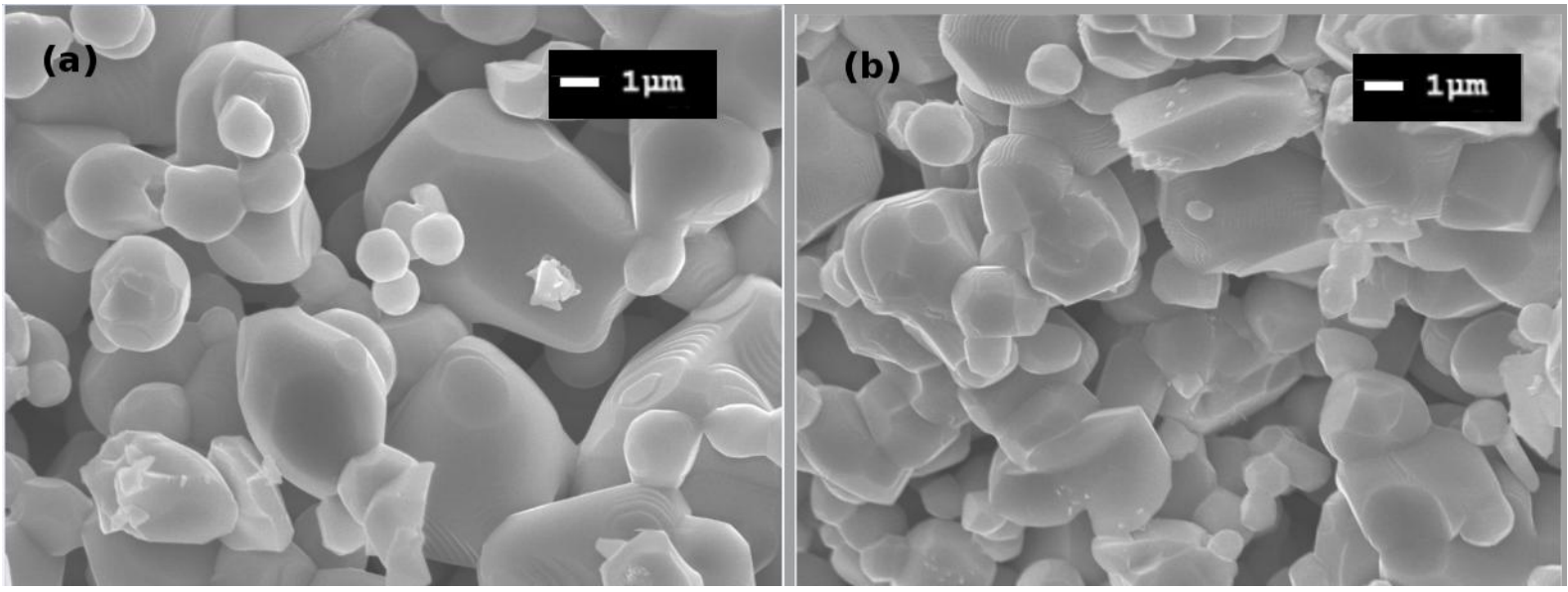

Figure 1

18

19

20

21

22

23

24

25

26

27

28

29

30

31

32

33

34

35

36

37

38

39

40

41

42

43

44

45

46

47

48

49

50

51

52

53

54

55

56

57

58

59

60

61

62

63

64

65 

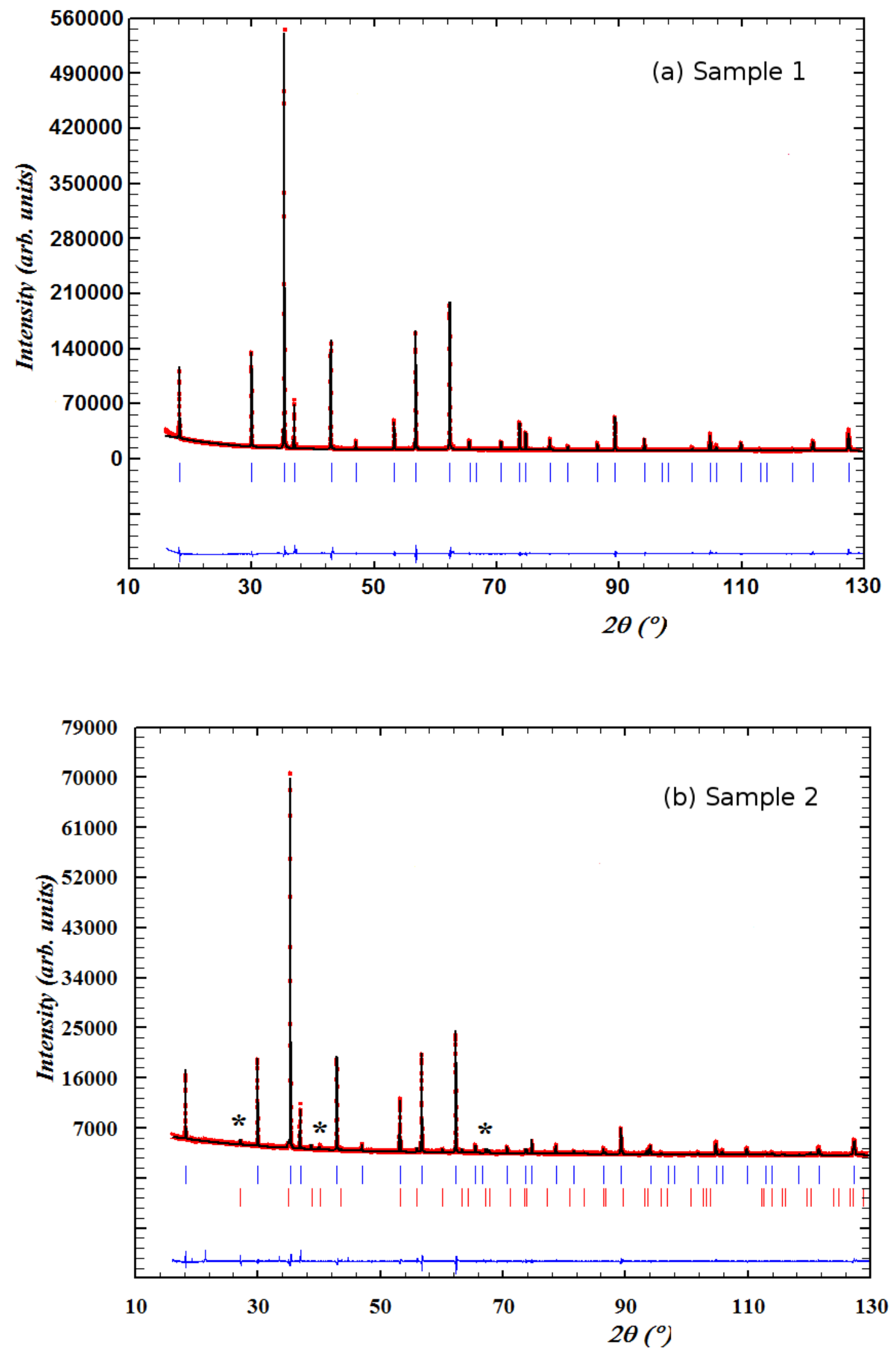
Figure 2

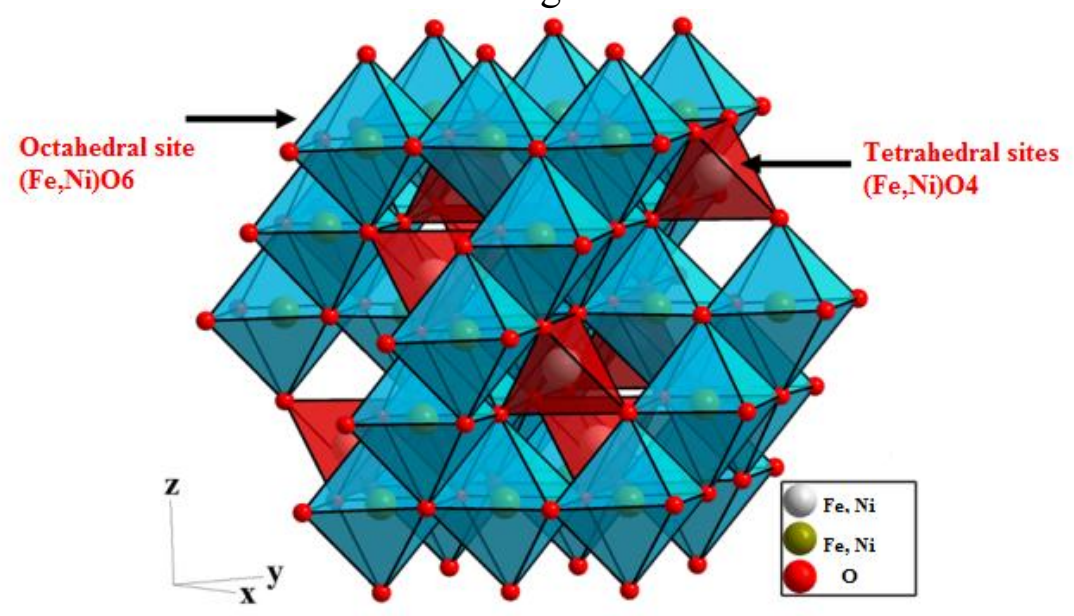

Figure 3 
1

2
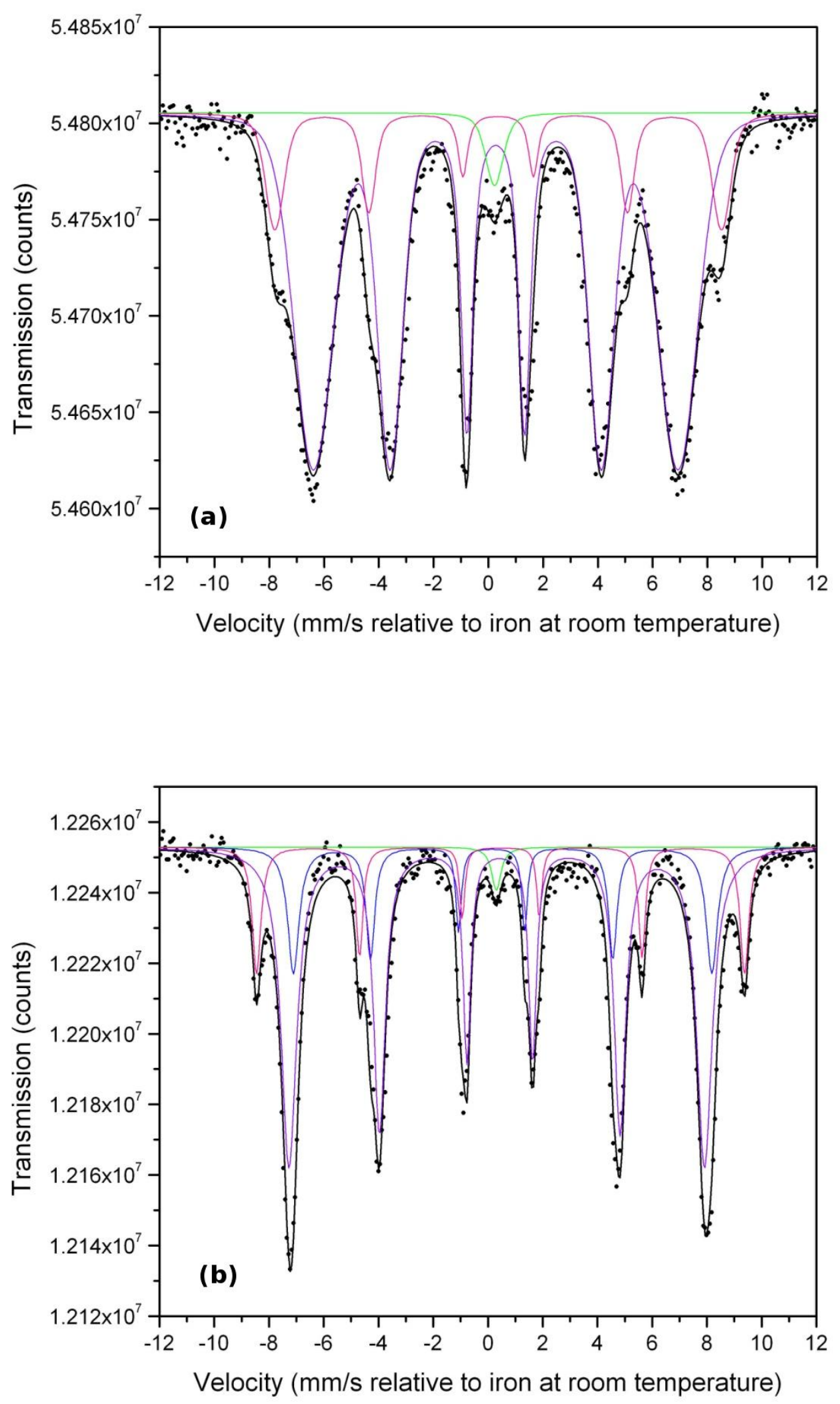

Figure 4 


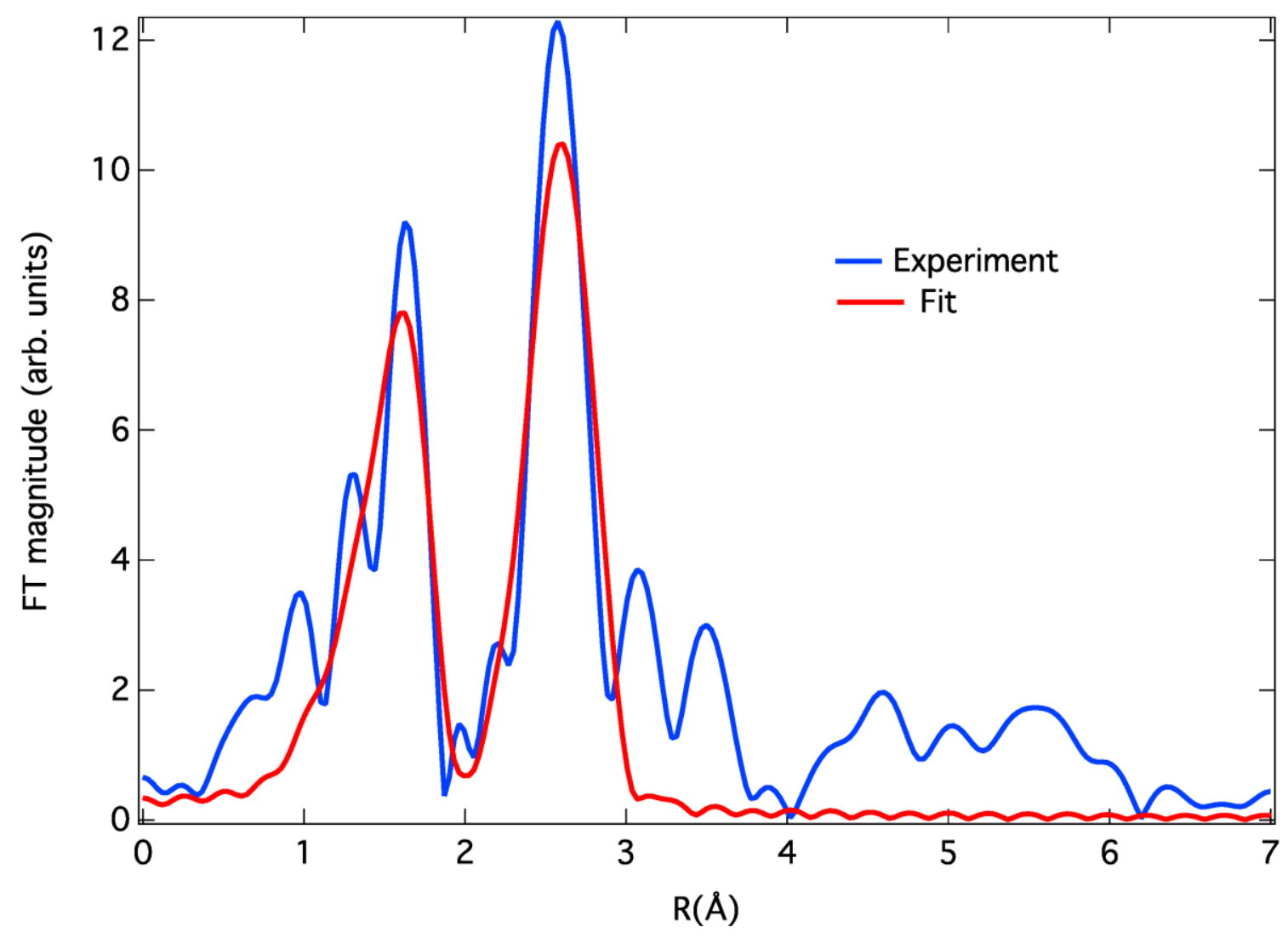

Figure 5 

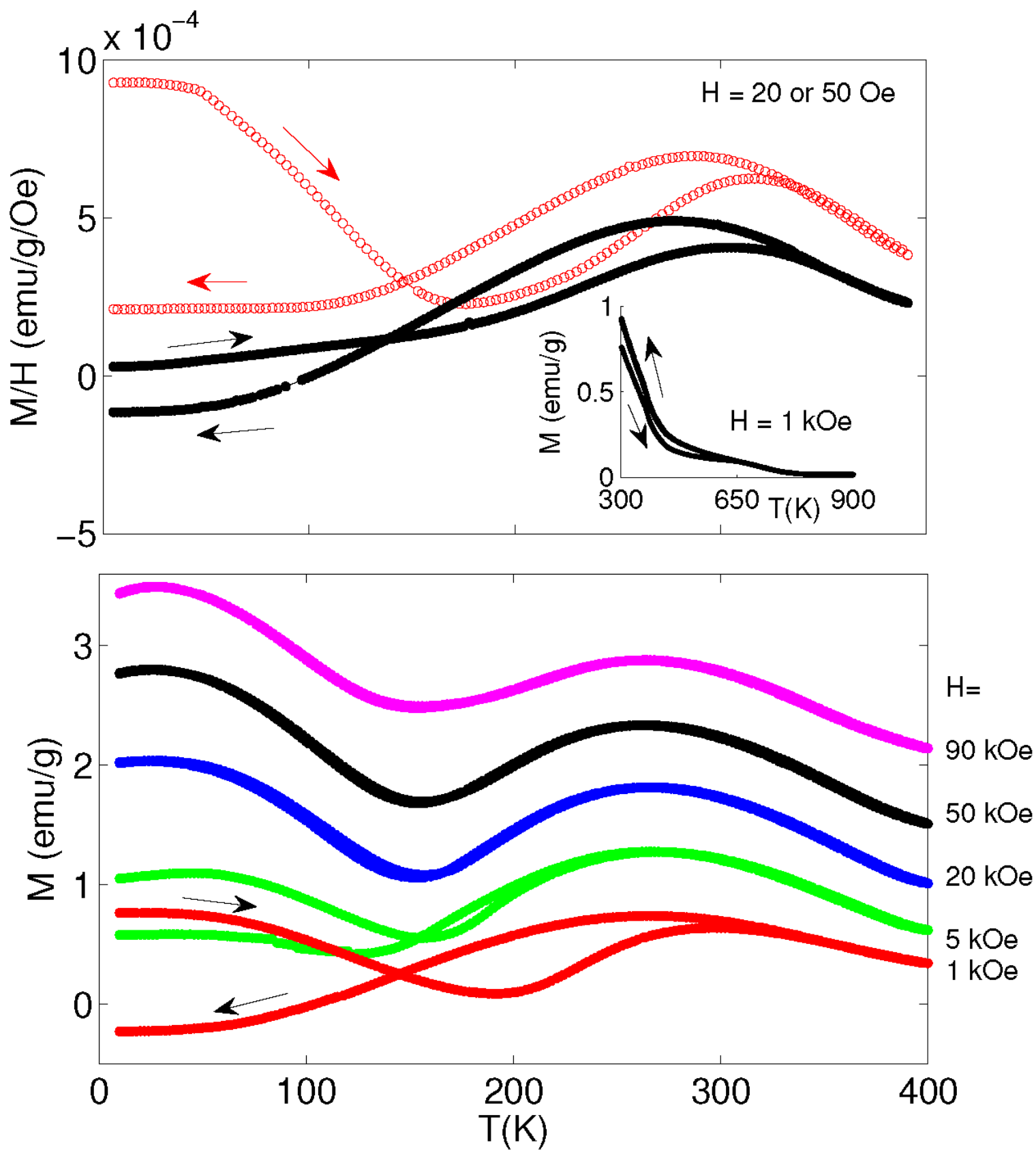

Figure 6 

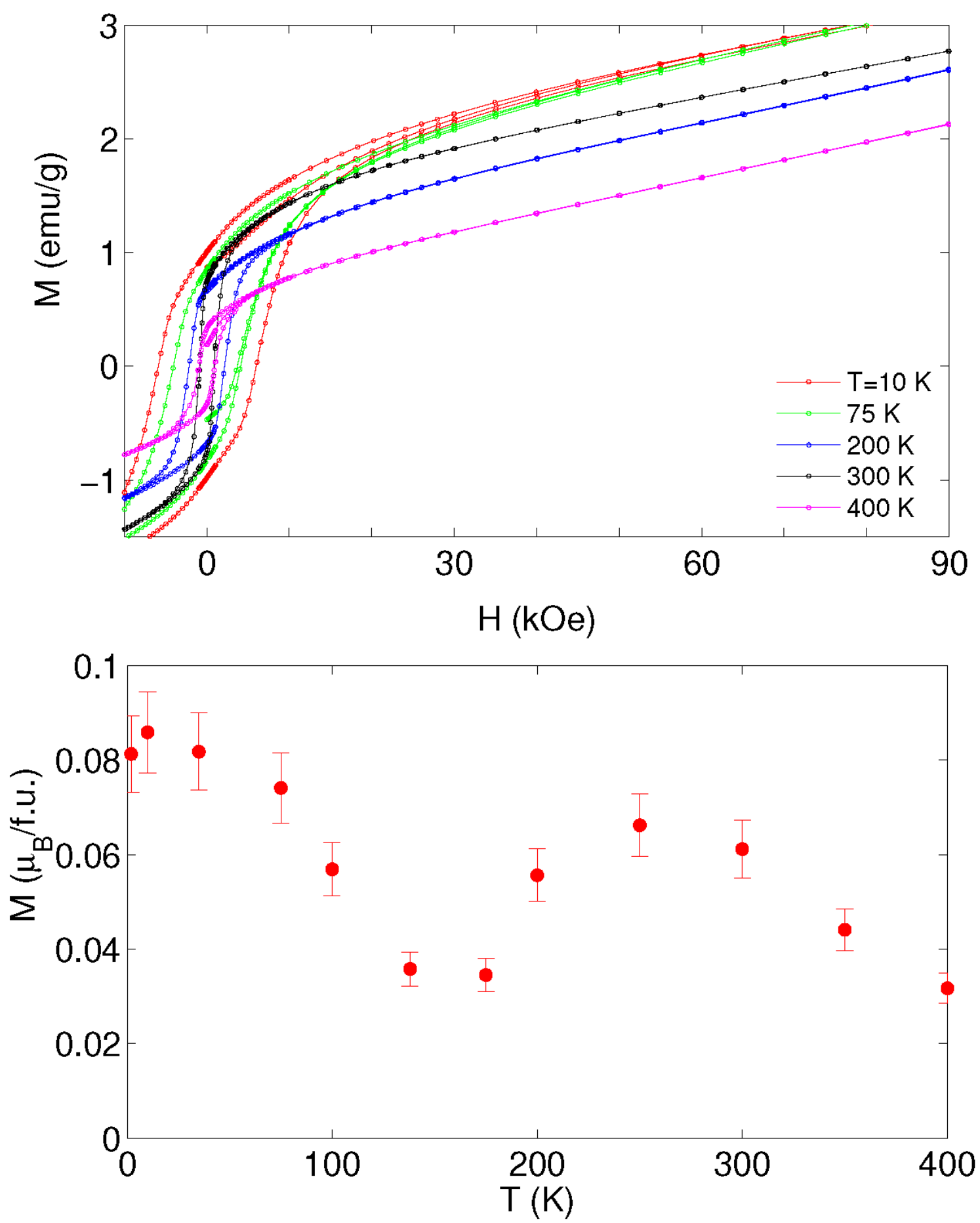

Figure 7 


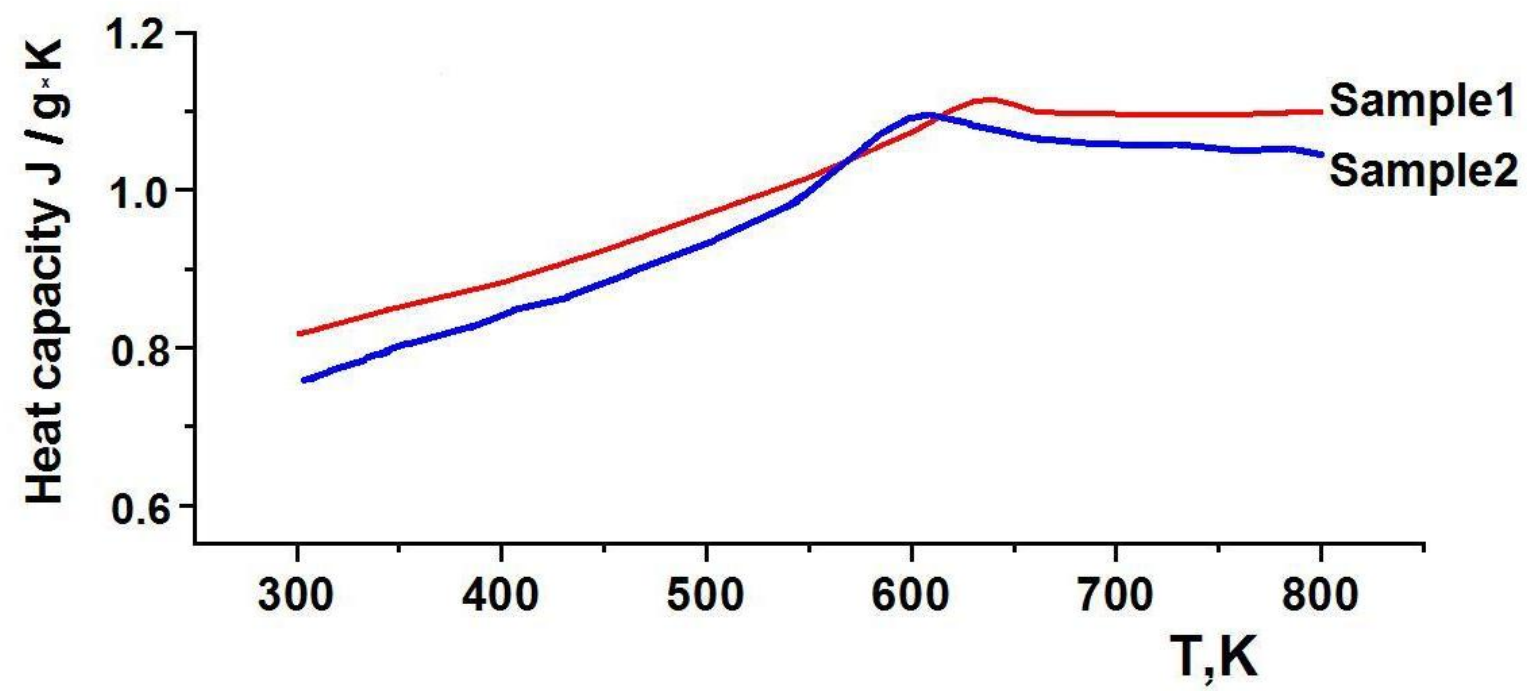

Figure 8

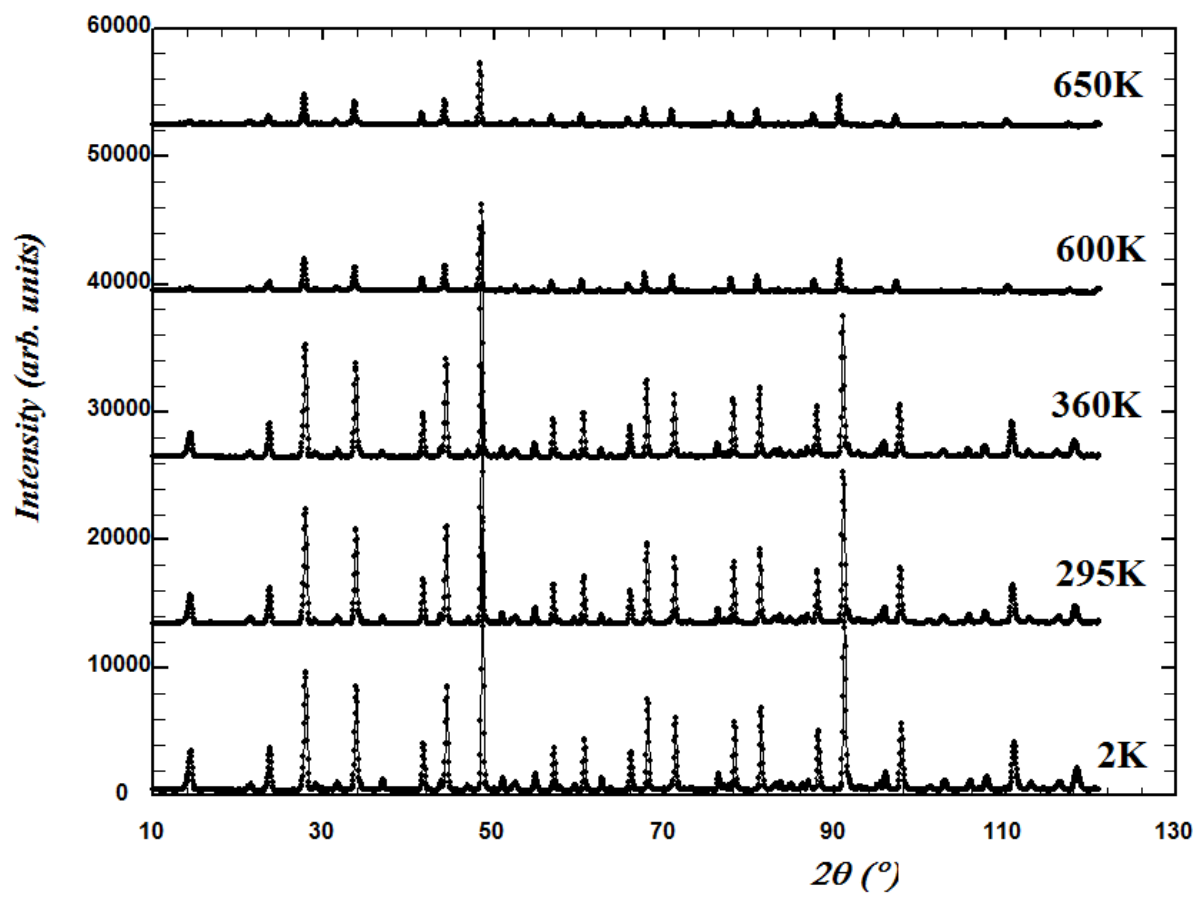

Figure 9 


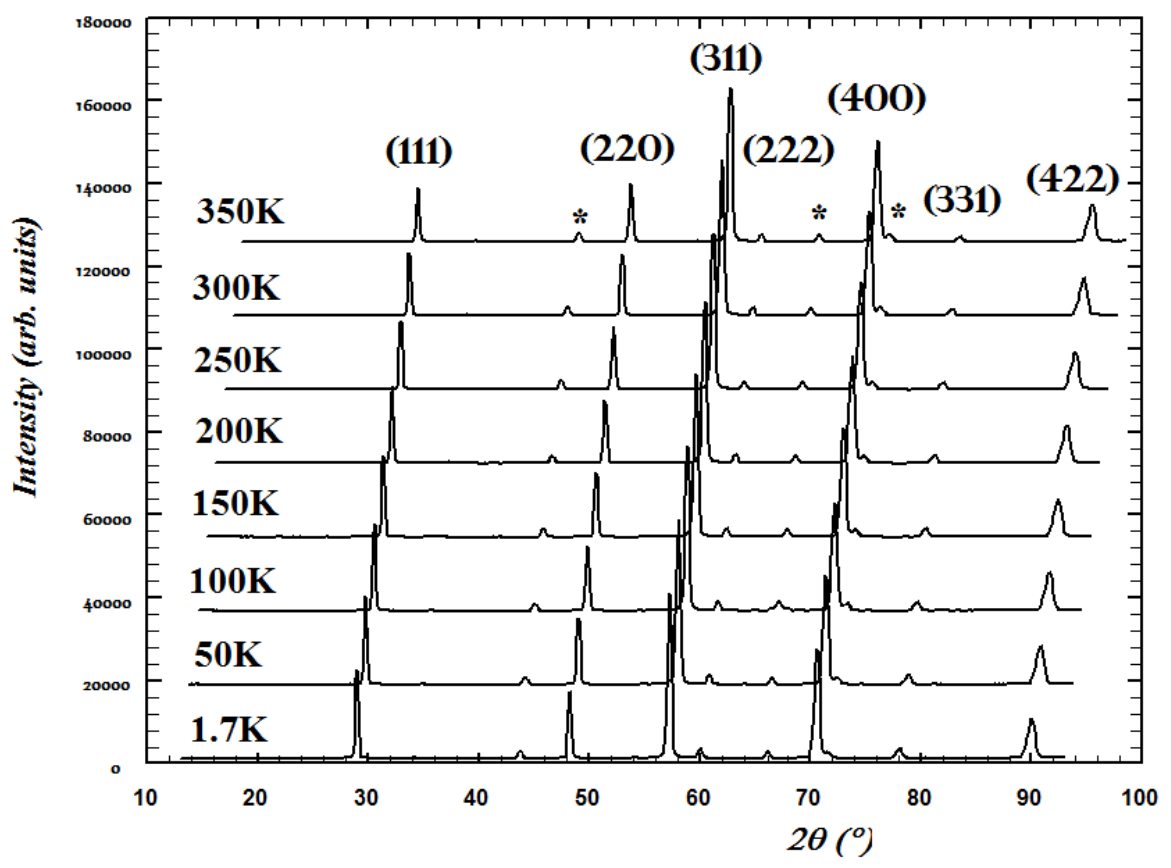

Figure 10 

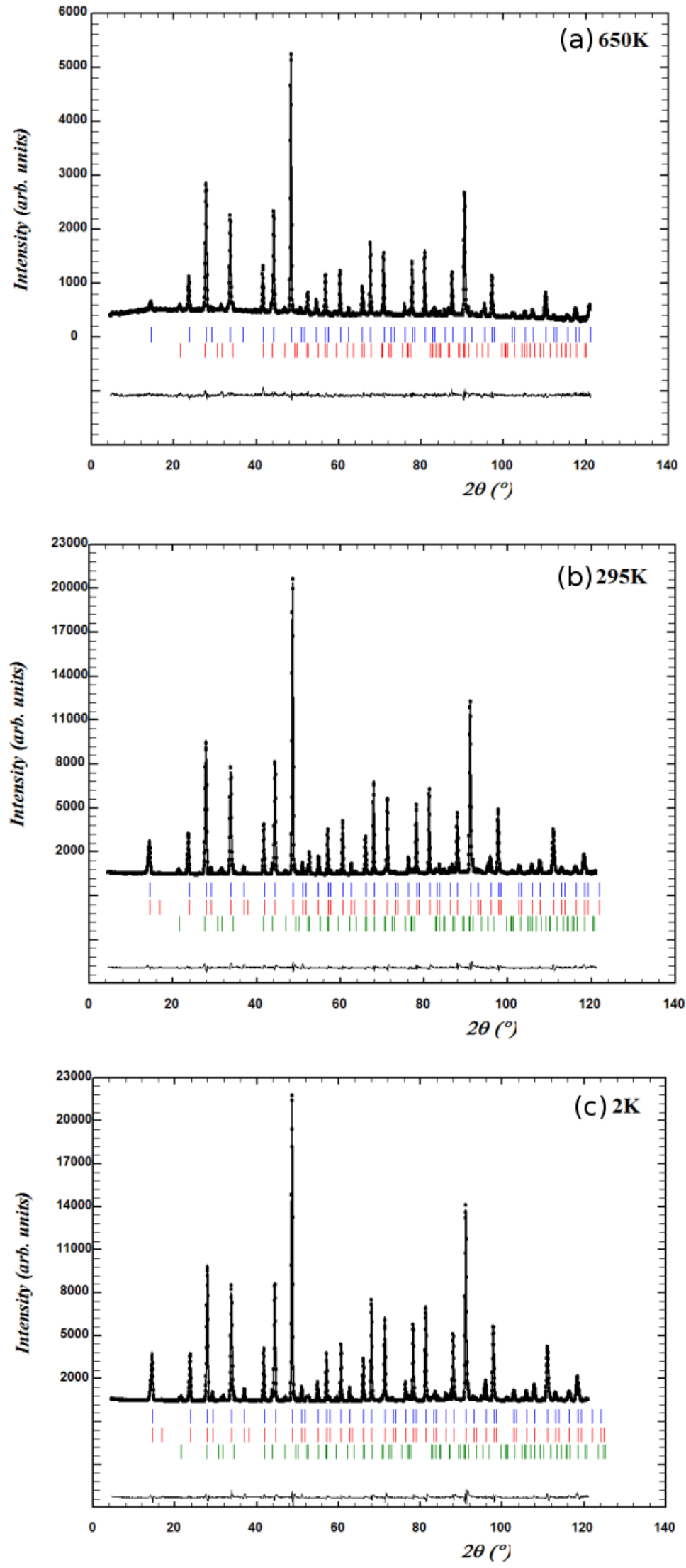

Figure 11 


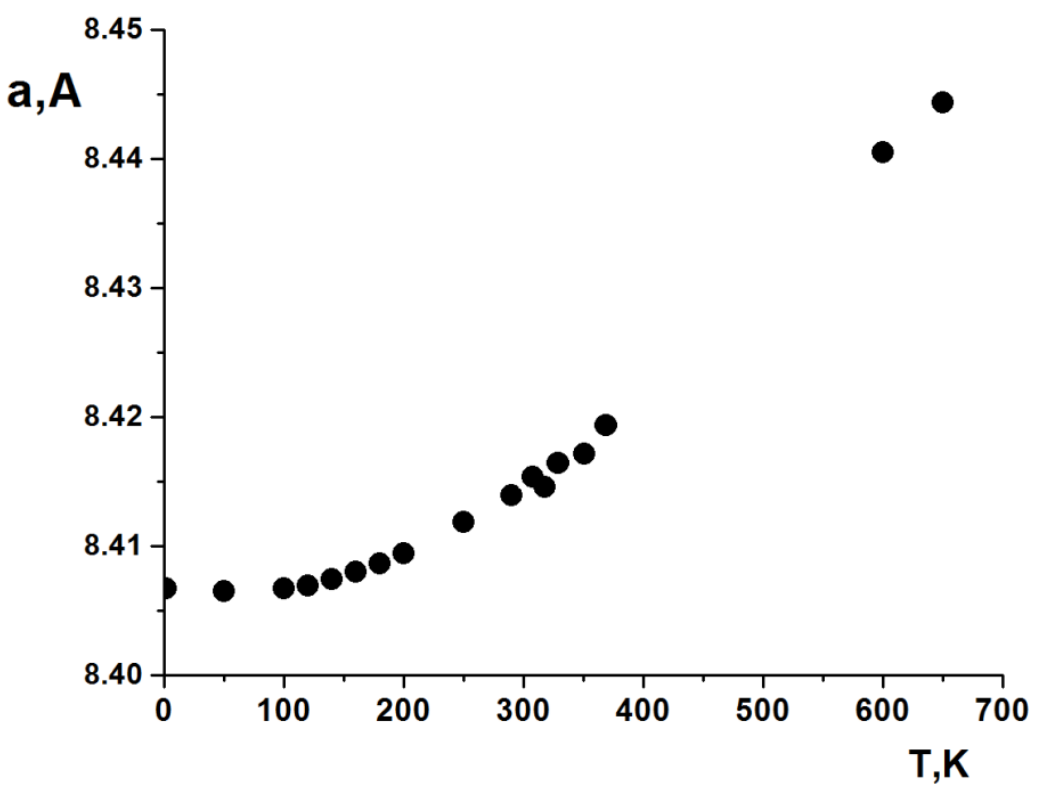

Figure 12 


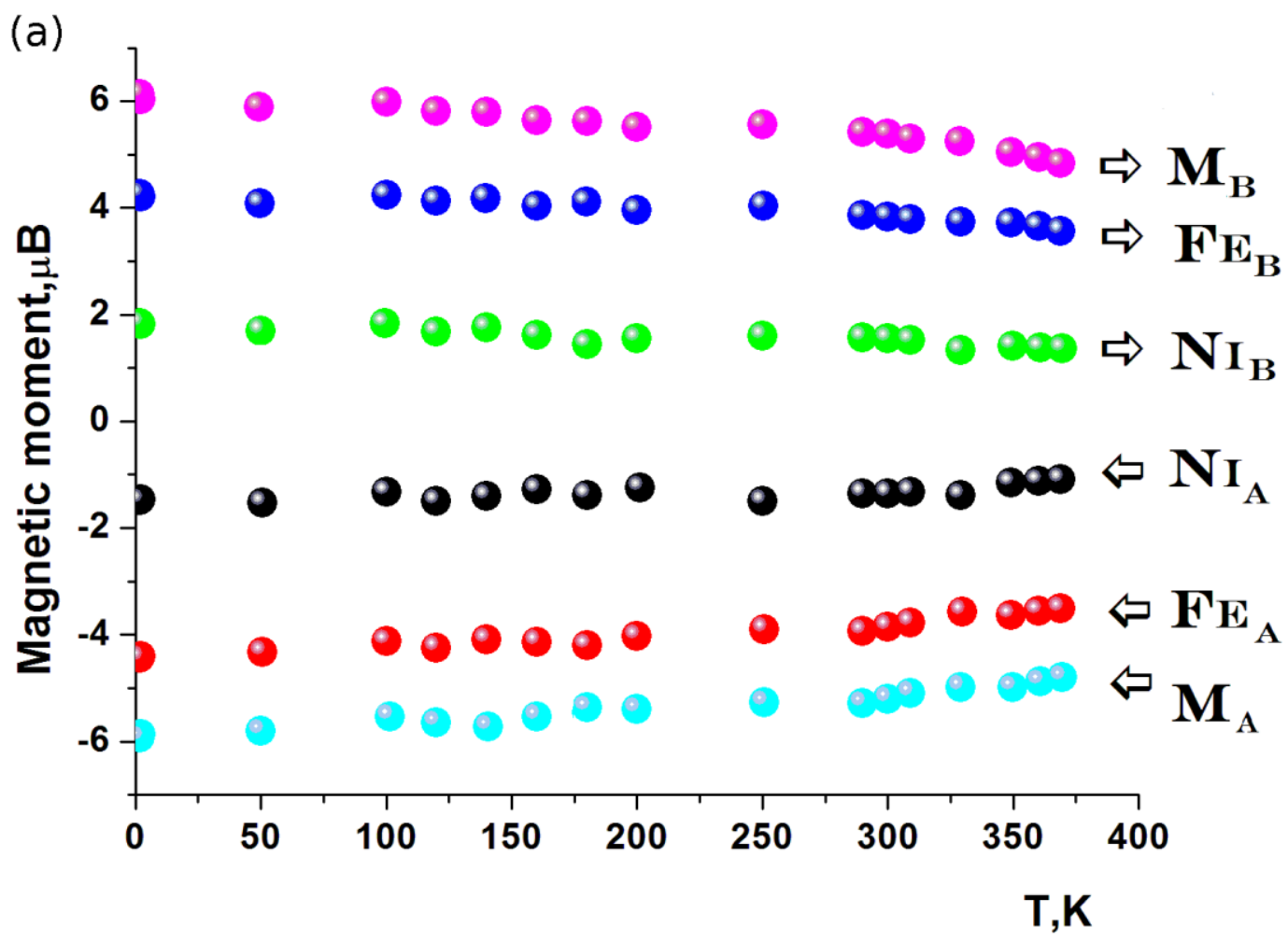

(b)

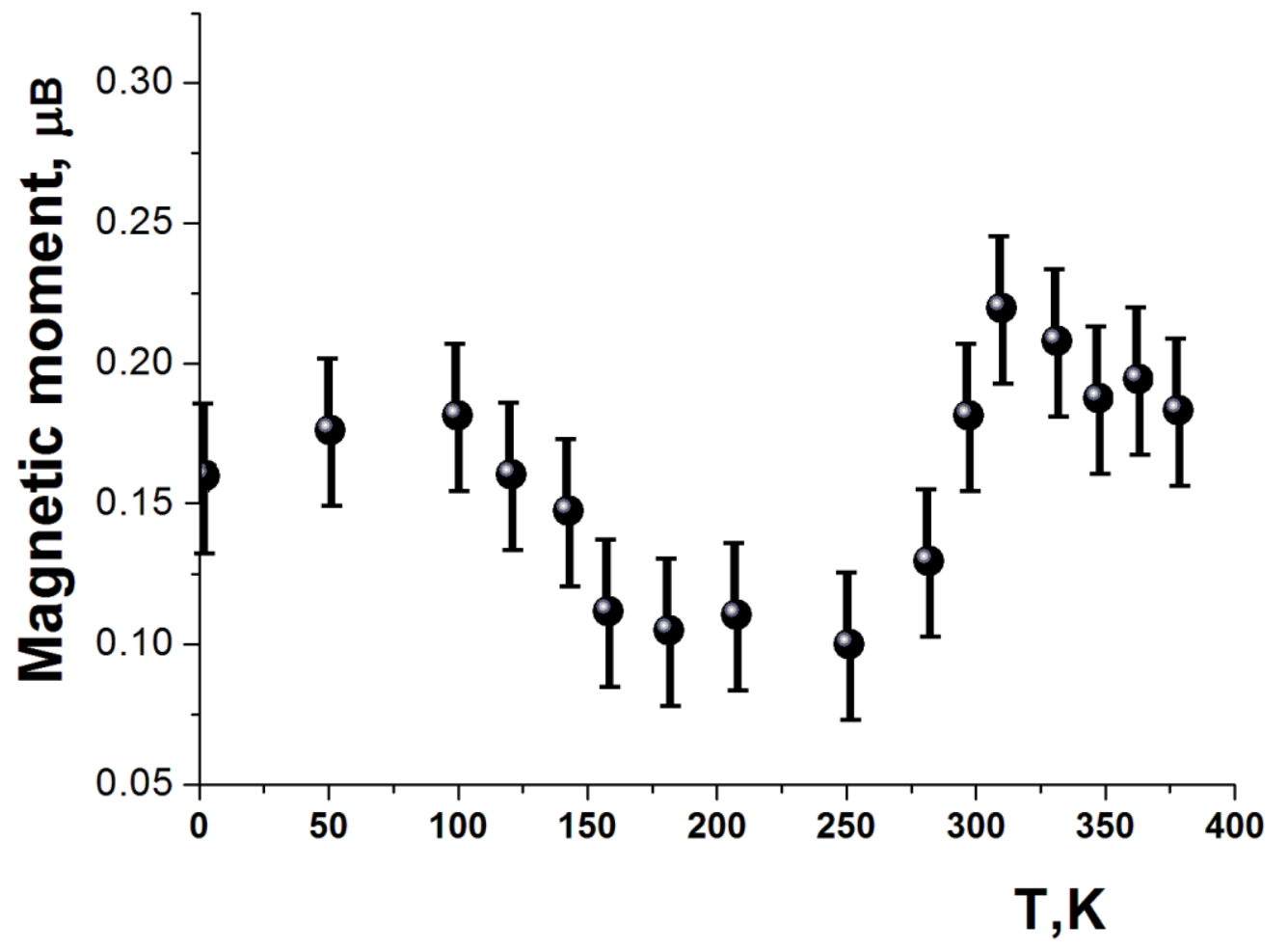

Figure 13 